\title{
Price competition in a nonlinear differentiated duopoly
}

\author{
Luca Gori*• Mauro Sodini ${ }^{\dagger}$
}

August 30, 2017

\begin{abstract}
This article represents an attempt to characterise the dynamics of a nonlinear duopoly with price competition and horizontal product differentiation by accounting for nonnegativity constraints (on profits and the market demand). The model is set up by following the tradition led by Bischi et al. (1998), according to which players have limited information. The article shows several local and global phenomena of a two-dimensional discrete time system when the price demand elasticity varies. It also points out the differences from both a mathematical and economic point of views in terms of dynamic outcomes when the non-negativity constraints are not binding and when they are binding. This is done by combining mathematical techniques and simulation exercises.
\end{abstract}

Keywords Chaos; Local and global bifurcations; Price competition; Product differentiation

JEL Classification C62; D43; L13

\section{Introduction}

This article represents an attempt to characterise local and global dynamics in a nonlinear duopoly with price competition and horizontal differentiation. Particular attention is paid to the role played by non-negativity constraints on quantities and profits in determining long-term outcomes.

In both static and dynamic models of industrial economics and industrial organisation, scholars often avoid dealing with microeconomic settings where the marginal willingness to pay

${ }^{*}$ L. Gori (corresponding author), Department of Political Science, University of Genoa, Piazzale E. Brignole, 3a, I-16125 Genoa (GE), Italy, e-mail: luca.gori@unige.it or dr.luca.gori@gmail.com, tel.: +39010209 9503 , fax: +390102095536.

${ }^{\dagger}$ M. Sodini, Department of Economics and Management, University of Pisa, Via Cosimo Ridolfi, 10, I-56124 Pisa (PI), Italy, e-mail: mauro.sodini@unipi.it, tel.: +39 0502216 234, fax: +39 0502210603. 
of consumers is captured by non-linear inverse demand functions. The majority of contributions, in fact, was concerned with models characterised by linear demands. This assumption is essentially made up for analytical tractability as well as for preserving concavity in the firm's optimisation problem and the uniqueness of the equilibrium. However, from an empirical point of view, there exist robust estimations supporting the existence of (individual or market) inverse demand functions describing a non-linear course between price and quantity (Varian, 1982, 1990). This holds in models with both price competition (Bertrand) and quantity competition (Cournot). We recall that when products are homogeneous firms choose to compete on quantities (Singh and Vives, 1984). In fact, a classic result in the duopoly theory is that price competition with homogeneous products leads to the unrealistic Bertrand paradox, resulting in the zero profit condition for competitors. This result does no longer hold in the case of product differentiation. In fact, as noted by Puu (2017), in the latter case the two firms have a kind of monopoly power (monopolistic competition) and hence a price competition setting can also be compatible with the existence of a Nash equilibrium with positive profits. Even empirically, there are classic results that appear to confirm that on actual markets competition takes place on prices when products are differentiated. (Gasmi et al., 1992).

Given the importance of studying problems related to product differentiation, ${ }^{1}$ our goal is to analyse the dynamic properties of a two-dimensional discrete time model where firms compete on prices. As obtaining and using information efficiently (as predicted by the rational expectations paradigm) seems to be a too strong assumption which can lead to high costs (Brock and Hommes, 1997), we assume that players have limited information and use a naïve rule (bounded rationality) to set the price for the subsequent period. ${ }^{2}$ Roughly speaking, when bounded rationality is introduced in economic models results are somewhat different from those obtained under rational expectations, especially with regard to stability outcomes. In fact, in the absence of full information models predict that instability holds under weak conditions ${ }^{3}$ : the instability of equilibria seems to be the rule rather than the exception and may represent an explanation of the observed output fluctuations (quantity or price) in imperfectly competitive markets.

With specific regard to the duopoly dynamics, the literature has essentially concentrated on quantity-setting firms by assuming either linear demand (quadratic utility) or unit-elastic demand (Cobb-Douglas utility). In a leading article, Bischi et al. (1999) draw attention on

\footnotetext{
${ }^{1}$ See Singh and Vives (1984) for the case of profit-maximising firms and Fanti et al. (2017) for the case of managerial firms.

${ }^{2}$ Models with rational expectations are based on two main assumptions: rationality of agents (i.e., expectations of an economic agent are equivalent to mathematical expectations, which exploit all available information) and homogeneity of expectations of all economic agents.

${ }^{3}$ Rational expectations models tend to explain the volatility of economic variables because of the existence of exogenous (stochastic) shocks, while instability in models with bounded rationality is endogenous to the model. See Agliari, Chiarella and Gardini (2006) for a critique to the use of rational expectations in dynamic (nonlinear) models.
} 
the existence of heterogeneities between players (asymmetric map) that give rise to symmetrybreaking bifurcations. They show that negligible differences in the basic parameters characterising duopolistic firms may cause qualitatively different dynamic evolutions as compared with the case of a symmetric game (such as lack of synchronisation and coexistence of attractors), i.e. the representative agent hypothesis dramatically matters for the outcome the economy may follow. More recently, it has been pointed out that there may exist similar dynamic events also when players are homogeneous and then the system characterising the dynamics of the economy is symmetric (Fanti et al., 2012, 2015). Specifically, Fanti et al. (2012) develop a nonlinear duopoly model with linear demand and managerial firms to show that the degree of competition between managers is responsible for on-off intermittency, blow-out phenomena and multistability, whereas Fanti et al. (2015) consider a model with homogeneous products, general isoelastic demand and profit maximising firms finding that the elasticity of market demand is responsible for local and global outcomes that cannot be observed in the case of unit-elastic demand (coexistence of attractors, coordination failures and complex structures of the basins of attraction). In this literature, some exceptions that study price competition with limited information are the works of Fanti et al. (2013a), Ahmed et al. (2015) and Andaluz and Jarne (2016). The first work considers a horizontally differentiated duopoly with homogeneous firms and linear demand (symmetric map). The main aim is to show that the extent of product differentiation is responsible for synchronised dynamics along the invariant diagonal and intermittency. The work also discusses the transition from simple dynamics to complex dynamics and describes the structure of the attractors and their basins. The second article concentrates on the case of a differentiated duopoly with substitute goods where consumers' demands are defined by starting from a CES (Constant Elasticity of Substitution) utility function, showing how different parameter configurations can affect the dynamics of the system. The third work, instead, considers a vertically differentiated duopoly and compares local and global dynamics of Cournot and Bertrand competition models. However, and most importantly, these articles do not take into account non-negativity constraints on quantities and profits and their role in long-term outcomes.

The present work emphasises the importance of economic constraints in determining dynamic outcomes when price competing firms do not have complete information. Results are given by considering gradual reductions in a parameter that contributes to measure the demand elasticity. This reduction may produce an increase in both unitary profits and marginal profits that contributes to let the change of prices over time much more reactive (to capture the opportunity of higher profitability) than when the elasticity of market demand is lower. An increase in the reactivity of firms in setting the price between two subsequent periods is responsible for several phenomena (Neimark-Sacker bifurcations, multistability) that are impossible in the case of unit-price elasticity. With regard to the role of economic constraints, the introduction of a piecewise map that makes it feasible regions of the phase plan that were excluded from previous studies leads to changes in the dynamics of the system properties that have required a 
thoughtful analysis. In particular, new forms of multistability and synchronisation phenomena not observable in the absence of constraints may arise. From an economic point of view, it is possible to observe time series characterised by periods in which production is positive space out by periods without production or periods in which production is positive but leading to a very low mark up of the price per unit of good with respect to the marginal cost. This behaviour seems to mimic some of the phenomena observed in actual duopoly markets, as price competition is fiercer than quantity competition (Ellison and Snyder, 2014; Newbery and Greve, 2015).

In the literature, there are contributions that have introduced constraints on both the demand side and the supply side (no zero production) in nonlinear duopoly models. As far as the former group of works is concerned, we mention the article of Bischi and Lamantia (2012) showing, in the basic Cournot model with linear reaction functions, that the existence of constraints on the size of market demand can generate routes to complex dynamics by starting from a situation where the Nash equilibrium is positive but unstable in the absence of constraints. As far as the second group of works is concerned, the literature (Agliari, Gardini and Puu, 2006; Tramontana et al., 2010) focuses on models with quantity competition and perfect knowledge of the market demand (best reply). In particular, Agliari, Gardini and Puu (2006) emphasise the possibility of obtaining multistability (attractors coexisting with a stable Nash equilibrium) due to constraints on the supply side of the market. Finally, with the aim of avoiding the unpleasant result that once a firm chooses to do not produce it definitely exits the market, Tramontana et al. (2010) introduce a lower bound on the amount produced by each single firm. Our price-setting mechanism resembles this assumption. However, different from Tramontana et al. (2010) in the present work it is possible to obtain that the quantity demanded by customers corresponding to a certain price at some time is null (with the opportunity to re-enter to the market later). This is because competition (firms' decisions) takes place on prices and market demand is the result of such decisions.

The rest of the article is organised as follows. Section 2 develops a Bertrand game with horizontal product differentiation in a standard static set up. Section 3 introduces a dynamic mechanism of prices by assuming limited information as in Bischi et al. (1998) and shows that reducing (ceteris paribus) the demand elasticity causes several local and global phenomena (Neimark-Sacker bifurcation, multistability and so on) that cannot be observed in a model with unit-elastic demand. Section 4 explores the role of economic non-negativity constraints by adopting the critical curves technique. Section 5 outlines the conclusions.

\section{The game}

Consider a Bertrand duopoly with horizontal product differentiation and two types of agents: firms and consumers. Firm 1 produces output of variety $1\left(q_{1} \geq 0\right)$ and firm 2 produces output 
of variety $2\left(q_{2} \geq 0\right)$. We assume that the indirect demand of product $i=1,2$ takes the following form:

$$
p_{i}=Q_{i}^{\frac{-1}{\eta}}
$$

where $Q_{i}=q_{i}+d q_{-i},-i$ identifies the rival firm of $i, \eta>0$ is a parameter that contributes to measure the degree of demand elasticity, $p_{i} \geq 0$ is the consumers' marginal willingness to pay towards product of variety $i$ produced by firm $i$ and $d$ is the degree of horizontal product differentiation. The demand function in (1) modifies the general isoelastic demand function of Fanti et al. (2015) by considering horizontally differentiated products. The direct demand of product of variety $i$ is then given by:

$$
q_{i}=\max \left(0, \frac{p_{i}^{-\eta}-d p_{-i}^{-\eta}}{1-d^{2}}\right) .
$$

If $q_{i}>0,(2)$ can be rewritten as follows:

$$
q_{i}=\frac{p_{i}^{-\eta}-d p_{-i}^{-\eta}}{1-d^{2}}=\frac{p_{i}^{-\eta}}{1-d^{2}}-\frac{d p_{-i}^{-\eta}}{1-d^{2}}
$$

from which it is possible to note that product $i$ is an ordinary good if and only if $d \in(-1,1)$. In particular, products are substitutes (resp. complements) if $d>0$ (resp. $d<0$ ), whereas they tend to be perfect substitutes (resp. perfect complements) if $d \rightarrow 1$ (resp. $d \rightarrow-1$ ). When $d<0$ direct demands of products 1 and 2 are both always positive. In contrast, when $d>0$ we have that $q_{i}>0, i=1,2 \Longleftrightarrow p_{-i} \in\left(d^{\frac{1}{\eta}} p_{i}, d^{-\frac{1}{\eta}} p_{i}\right)$. This non-negativity condition plays a relevant role in our model, as will be clear later in this article.

The production function of firm $i$ has constant marginal returns to labour implying that $q_{i}=L_{i}$, where $L_{i}$ is the labour force employed in $i$ th firm (Correa-López and Naylor, 2004). Then, firm $i$ 's cost function is $W_{i}\left(q_{i}\right)=w L_{i}=w q_{i}$, where $w>0$ is the constant average and marginal cost (wage) of producing an additional unit of output. Firm $i$ maximises profits $\Pi_{i}=\max \left(0, p_{i}-w\right) \max \left(0, q_{i}\right)$ with respect to $p_{i}$, given $p_{-i}$ and the parameters of the model. 1) If $w>d^{\frac{-1}{\eta}} p_{-i}$ then it is not possible for firm $i$ to realise positive profits. In such a case, the objective of firm $i$ is to avoid negative profits. Therefore, there exist two optimal solutions of the price-setting problem for firm $i$. The first one is $p_{i}=w$, implying that irrespective of the market demand the profit of firm $i$ is null. The second one is $p_{i}>d^{\frac{-1}{\eta}} p_{-i}$, implying that the price is fixed at too high a level so that the demand of product of firm $i$ is zero. 2) It is simple to verify that if $w<d^{\frac{-1}{\eta}} p_{-i}$ then the solution of the optimisation problem of firm $i$ is a price $p_{i}$ such that the demand $q_{i}$ and profits $\Pi_{i}$ are positive. In this case, the optimisation problem of firm $i$ can be written as follows:

$$
\max _{w<p_{i}<d^{\frac{-1}{\eta}} p_{-i}}\left(p_{i}-w\right)\left(\frac{p_{i}^{-\eta}-d p_{-i}^{-\eta}}{1-d^{2}}\right) .
$$

By computing marginal profits 


$$
\frac{\partial \Pi_{i}}{\partial p_{i}}=\frac{p_{i}^{1-\eta}(1-\eta)+p_{i}^{-\eta} \eta w-d p_{i} p_{-i}^{-\eta}}{\left(1-d^{2}\right) p_{i}}
$$

we have that

$$
\operatorname{sgn}\left(\frac{\partial \Pi_{i}}{\partial p_{i}}\right)=\operatorname{sgn}\left(x_{i}\left(p_{i}\right)\right)
$$

where

$$
x_{i}\left(p_{i}\right):=(1-\eta) p_{i}^{-\eta}+\eta w p_{i}^{-\eta-1}-d p_{-i}^{-\eta} .
$$

From (7) we get

$$
\lim _{p_{i} \rightarrow d^{\frac{-1}{\eta}} p_{-i}} x_{i}\left(p_{i}\right)=-\eta d p_{-i}^{-\eta}\left[1-\frac{w}{d^{\frac{-1}{\eta}} p_{-i}}\right]<0
$$

and

$$
\lim _{p_{i} \rightarrow 0^{+}} x_{i}\left(p_{i}\right)=+\infty
$$

We have that the derivative of $x_{i}$ with respect to $p_{i}$ is given by $x_{i}^{\prime}\left(p_{i}\right)=-\eta p_{i}^{-\eta-1}\left[1-\eta+\frac{(1+\eta) w}{p_{i}}\right]$. If $0<\eta<1$ then $x_{i}^{\prime}\left(p_{i}\right)<0$. Thus, $x_{i}\left(p_{i}\right)$ is a monotonically decreasing function. If $\eta>1$ then we have that function $x_{i}\left(p_{i}\right)$ is first decreasing and then increasing. Therefore, in any case there exists a unique point $\bar{p}_{i}$ such that $\partial \Pi_{i} / \partial p_{i}>0$ for any $p_{i}<\bar{p}_{i}$ and $\partial \Pi_{i} / \partial p_{i}<0$ for any $p_{i}>\bar{p}_{i}$. As a consequence, $\bar{p}_{i}$ is the solution of the problem of firm $i$.

Equilibria of the game. Let us now concentrate on the solution of the static game. We begin the study by showing that $(w, w)$ is not a Nash equilibrium of the game. Assume that player 2 plays $w$. Then, we will show that player 1 should play a price such that $p_{1}>w$. Given the assumptions on the behaviour of player 2, we have that

$$
\Pi_{1}=\left(p_{1}-w\right) \max \left(0, \frac{p_{1}^{-\eta}-d w^{-\eta}}{1-d^{2}}\right) .
$$

If firm 1 played $w$ it would get a payoff $\Pi_{1}=0$, but we now show the existence of a price $p_{1}$ that verifies system

$$
\left\{\begin{array}{c}
p_{1}^{-\eta}-d w^{-\eta}>0 \\
p_{1}>w
\end{array},\right.
$$

and guarantees strictly positive profits to firm 1. For doing this, we have to distinguish between two cases: product complementarity ( $d<0$, that is prices are strategic substitutes) and product substitutability ( $d>0$, that is prices are strategic complements). Then, in order to get positive profits it is sufficient to set $p_{1}>w$ when $d<0$ and $w<p_{1}<d^{\frac{-1}{\eta}} w$ when $d>0$.

We proceed further into the analysis by showing that Nash equilibrium $\left(p_{1}^{0}, w\right)$, with $p_{1}^{0}>w$, cannot exist for the game. Specifically, we will see that given $p_{1}^{0}$ player 2 should not play $w$. From the arguments proposed in the solution of the optimisation problem it is sufficient to prove that there exists a price $p_{2}>w$ such that

$$
q_{2}=\frac{p_{2}^{-\eta}-d\left(p_{1}^{0}\right)^{-\eta}}{1-d^{2}}>0
$$


If fact, this strategy guarantees strictly positive profits for player 2 . In particular, if $d<0$ then condition (12) is fulfilled for any $p_{2}>w$, whereas if $d>0$ it is sufficient to set $p_{2}$ such that $w<p_{2}<d^{\frac{-1}{\eta}} p_{1}^{0}$ so that condition (12) holds.

By concentrating on the existence of Nash equilibria such that prices of both firms are strictly larger than $w$ with $p_{-i} \in\left(d^{\frac{1}{n}} p_{i}, d^{-\frac{1}{\eta}} p_{i}\right), i=1,2$, we find that the optimality conditions for interior points (first order conditions) imply that the equations

$$
(1-\eta) p_{i}^{1-\eta}+\eta w p_{i}^{-\eta}-d p_{i} p_{-i}^{-\eta}=0, \quad i=1,2,
$$

should hold at Nash equilibrium $\left(p_{1}^{0}, p_{2}^{0}\right)$.

As the game is symmetric, there exists at least a symmetric equilibrium $\left(p^{0}, p^{0}\right)$, where $p^{0}$ is solution of the following equation:

$$
\left(p^{0}\right)^{1-\eta}(d+\eta-1)-\left(p^{0}\right)^{-\eta} \eta w=0 .
$$

From (14), it follows that the Nash equilibrium is unique. This is given by:

$$
E^{*}:=\left(p_{1}^{*}, p_{2}^{*}\right)=\left(\frac{\eta w}{d+\eta-1}, \frac{\eta w}{d+\eta-1}\right) .
$$

Then, equilibrium values of quantities and profits are respectively given by:

$$
\begin{gathered}
q_{1}^{*}=\frac{\left(p_{1}^{*}\right)^{-\eta}}{1+d}, \quad q_{2}^{*}=\frac{\left(p_{2}^{*}\right)^{-\eta}}{1+d}, \\
\Pi_{1}^{*}=\frac{w(1-d)}{d+\eta-1} q_{1}^{*}, \quad \Pi_{2}^{*}=\frac{w(1-d)}{d+\eta-1} q_{2}^{*} .
\end{gathered}
$$

Remark 1 If $\eta>1$ then Nash equilibrium (15) is well defined for any $d>0$. However, the existence of a Nash equilibrium is not guaranteed for any couple $(d, \eta)$. In particular, if $\eta<1$ (i.e., the market demand is sufficiently elastic) then $d>1-\eta$ (i.e., products 1 and 2 should be sufficiently substitutes between each other), whereas if $d<0$ (i.e., products are complements) then $\eta>1-d>1$ (i.e., the market demand should be sufficiently anelastic for any given value of the degree of product differentiation).

In this article, we concentrate on the analysis of the local and global dynamics of the model when products are substitutes (i.e., prices are strategic complements) and the elasticity of market demand is relatively low ( $\eta$ is sufficiently high), implying that the degree of substitution 
between product of firm 1 (e.g., Coca-Cola) and product of firm 2 (e.g., Pepsi Cola) is relatively small for consumers. ${ }^{4}$ Therefore,

Assumption $2 d>0$ and $\eta>1$.

We now show that under Assumption 2 non-symmetric Nash equilibria do not exist.

Proposition 3 If a Nash equilibrium exists for the game, it is a symmetric equilibrium.

Proof. Let $\left(p_{1}^{0}, p_{2}^{0}\right)$ be a Nash equilibrium of the game. Define

$$
f(z)=\frac{[(1-\eta) z+\eta w]^{\frac{1}{\eta}}}{(d z)^{\frac{1}{\eta}}} .
$$

From the optimality conditions it follows that the condition:

$$
f\left(p_{2}^{0}\right)=\frac{p_{2}^{0}}{p_{1}^{0}}=\frac{1}{f\left(p_{1}^{0}\right)},
$$

must hold in equilibrium. By absurd, let us assume that $p_{1}^{0}>p_{2}^{0}$. Define also

$$
\tilde{f}(z)=\frac{(1-\eta) z+\eta w}{d z},
$$

which is a monotonically increasing transformation of $f(z)$. From (20) we have that the derivative of $f$ with respect to $z$ is given by

$$
\tilde{f}^{\prime}(z)=\frac{-\eta w d}{(d z)^{2}}<0,
$$

as $d>0$. Therefore, we get $f\left(p_{1}\right)<f\left(p_{2}\right)$ for any $p_{1}>p_{2}$. Then, equalities in (19) cannot hold.

Remark 4 When $d<0$ (i.e., products are complements and prices are strategic substitutes), it is possible to have both symmetric and non-symmetric equilibria.

\footnotetext{
${ }^{4}$ See Gasmi et al. (1992) for a study of a Bertrand soft drink market duopoly where products are substitutes and then prices and strategic complements (the reaction functions of firms are upward sloping). Empirical works less support the case in which products are complements so that prices and strategic substitutes (the reaction functions of firms are downward sloping). Then we do not present an analysis of such a case here. In a duopoly, two variables are said to be strategic complements (resp. substitutes) when a firm's action induces the rival to take the same (resp. opposite) action. These concepts help describing how a firm reacts to price/quantity change by a competitor to maximise profits.
} 


\section{Dynamics}

Time is discrete and indexed by $t=0,1,2, \ldots$ By following Bischi et al. (1998), we assume that each player has limited information about the market demand. In order to overcome this lacuna, player $i$ uses an adjustment mechanism based on the local estimate of its own marginal profits at time $t\left(\partial \Pi_{i} / \partial p_{i}\right)$ to set the price for time $t+1$. By abstracting from any possible non-negativity (economic) constraint, the adjustment mechanism of firms in a framework where the price is the strategic variable reads as follows (gradient dynamics):

$$
p_{i}^{\prime}=p_{i}+\alpha p_{i} \frac{\partial \Pi_{i}}{\partial p_{i}}
$$

where ' is the unit-time advancement operator, $\alpha>0$ is the speed of adjustment of firm $i$ 's price and $\partial \Pi_{i} / \partial p_{i}$ are determined by (5). Although players have limited information, they are assumed to be able to get a local estimate of marginal profits in the current period. Therefore, firm $i$ increases (resp. decreases) its price at time $t+1$ if $\partial \Pi_{i} / \partial p_{i}>0\left(\operatorname{resp} . \partial \Pi_{i} / \partial p_{i}<0\right)$ at time $t$. Eq. (22) is a Bischi-like gradient rule applied to a model with price competition, where we have used the linear specification $\alpha\left(p_{i}\right)=\alpha p_{i}$. This means that a relative change in price from time $t$ to time $t+1$, i.e. $\frac{p_{i}^{\prime}-p_{i}}{p_{i}}$, is proportional to firm $i$ 's marginal profitability. Some clarifications are now in order about differences between models of price competition and models of quantity competition (such as the pioneering contribution of Bischi et al. and related works) using this kind of adjustment mechanism. Starting right from Bischi et al. (1998), we recall that the quantity adjustment mechanism proposed by the authors is related to the following economic framework: each duopolistic firm does not know the quantity produced by the rival and does not know market demand. Therefore, as technology requires one period of time to bring the production process to completion and get products to the market (time-tobuild assumption), based on this mechanism, production is started at time $t$ and the quantities chosen by the firm will be available to the market at time $t+1$. Now, based on the quantities produced by both firms consumers outline the market demand that was unknown to firms at time $t$. Differently, in a price competition model with a time-to-build technology, the economic framework changes. In particular, one should consider a market where firms offer a price for a good that once ordered by customers requires a time lag in production (for example, the car market). In this case, production decisions and the market demand at time $t+1$ are revealed at time $t$. It is interesting to note that a literature has recently emerged about the study of nonlinear duopoly dynamics resulting from the assumption (exogenous choice) that a firm is a price setter and the rival a quantity setter (e.g., Naimzada and Tramontana, 2012; Wang and Ma, 2014). However, given the above reasoning, the Cournot-Bertrand comparison in a dynamic framework with bounded rationality may cause problems of time consistency between the behaviour of agents on the demand side and the behaviour of agents on the supply side. Therefore, it requires a deeper investigation and understanding of the modelling approach.

In the literature on nonlinear duopolies, analyses have often been restricted to the study of 
dynamic systems generated by equations similar to (22) on set

$$
D=\left\{p_{1}, p_{2}: p_{1} \geq 0, p_{2} \geq 0\right\}
$$

without accounting for problems related to the existence of economic constraints (Fanti et al., 2013a). However, these constraints (positivity of quantities, non-negativity of profits, etc.) play a relevant role in the study of dynamic models in both cases of quantity competition and price competition duopolies. In fact, the inclusion of non-negativity constraints may change some (global) dynamic properties of the systems in comparison with the same models without constraints, as is stressed, for instance, by Cánovas et al. (2008) and Fanti et al. (2013b) for Cournot rivalry. Given these observations, in order to have an economically well-defined map we have to consider non-negativity constraints to account for 1) the non-negativity of quantities $\left(q_{i} \geq 0\right)$, and 2$)$ the condition that guarantees the non-negativity of profits. With specific regard to this last point, we note that profits of firm $i$ are given by $\Pi_{i}=\left(p_{i}-w\right) q_{i}$. As the firm's strategic variable is the price and given both the hypothesis of constant marginal returns to labour and the non-negativity of quantities, non-negativity of profits is guaranteed by setting the price at a value larger than or equal to the average and marginal cost $w$. Therefore, even though in this work we are assuming players with bounded rationality, at every date neither firm 1 nor firm 2 wants to set $p_{i}<w, i=1,2$. Then, we let $\bar{w}=w+\varepsilon(\varepsilon>0)$ be a lower bound on the price of both products, representing a sort of "epsilon" stand-by price (mark up) to avoid that firms voluntarily choose a price that leads to a zero profit condition (see Tramontana et al., 2010 for the use of this kind of threshold in a Cournot model where players have full information and naïve expectations). Parameter $\varepsilon$ can be interpreted as follows. In a dynamic context, firms prefer to set a precautionary price that will guarantee them non-negative profits even in the case of small shocks on the cost side. The existence of a condition that guarantees the nonnegativity of profits makes a nonlinear Bertrand duopoly sharply different than a nonlinear Cournot duopoly (where $q_{i} \geq 0$ always holds). In fact, in the latter kind of models where dynamics of quantities are driven by an adjustment mechanism à la Bischi et al. (1998), it is possible to have periods in which the profit is strictly negative. This is because the marginal willingness to pay of consumers at time $t$ is actually affected by decisions made by each firm at time $t-1$ on the basis of the behaviour of the rival at this last time. Indeed, we recall that $p_{i}^{\prime}=f\left(q_{i}^{\prime}, q_{-i}^{\prime}\right)$, where the quantities $q_{i}^{\prime}$ and $q_{-i}^{\prime}$ are chosen independently by each firm at time $t$ in a Cournot model, so that $p_{i}^{\prime} \gtrless w, i=1,2$.

By turning on to the study of a Bertrand model, we now restrict the analysis to set $F$ given by

$$
F=\left\{\left(p_{1}, p_{2}\right) \in A: T^{n}\left(p_{1}, p_{2}\right) \in A, \forall n \geq 0\right\}
$$

where $A$ is defined as

$$
A=\left\{\left(p_{1}, p_{2}\right) \in R^{2}: p_{1} \geq \bar{w}, p_{2} \geq \bar{w}\right\} .
$$

Now, given set $A$ it is possible to identify two different regions: 1) region $A \cap B$ where both 
firms produce positive quantities and $B$ is equal to

$$
B=\left\{\left(p_{1}, p_{2}\right) \in R^{2}: d^{\frac{1}{\eta}} p_{1}<p_{2}<d^{-\frac{1}{\eta}} p_{1}\right\}
$$

and 2) region $A \backslash B$ where one of the two firms does not produce positive quantities (see Figure $1)$. Specifically, set $B$ defined in (26) represents the set of prices such that quantities are nonnegative (see Eq. 3), whereas set $A$ defined in (25) represents the set of prices such that profits are non-negative. Set $A$ can actually be considered as the set of initial conditions such that the dynamics are well-defined and the set in which the behaviour at time $t$ of both players should be defined in such a way that the price at time $t+1$ is fixed.

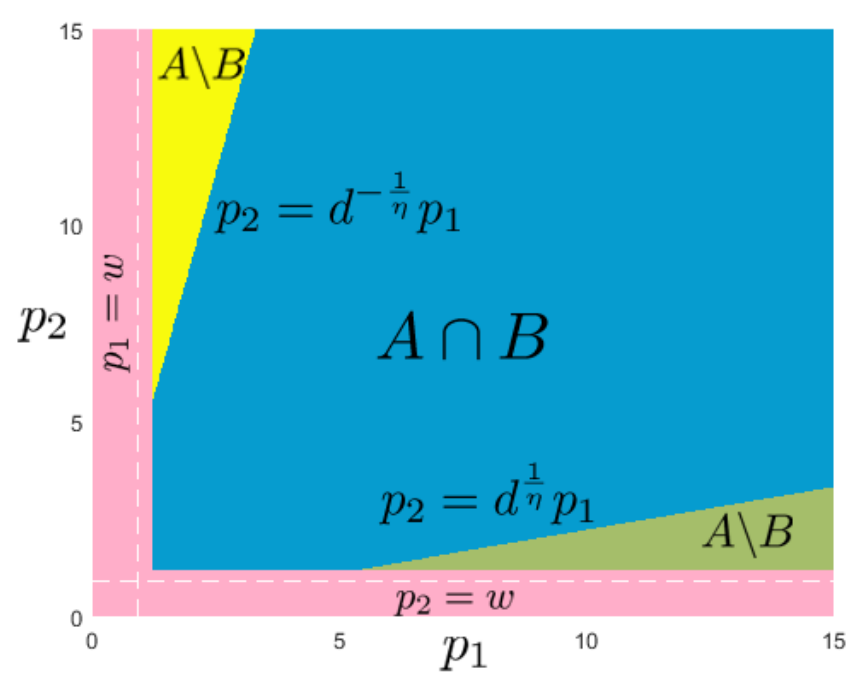

Figure 1. The blue region (bounded by the straight lines $p_{2}=d^{-\frac{1}{\eta}} p_{1}$ and $p_{2}=d^{\frac{1}{\eta}} p_{1}$ ) shows the set in which prices guarantees that both firms produce a positive quantity of both products. The yellow (resp. green) area describes a portion of the region in which the combination of prices such that the quantity produced by firm 2 (resp. firm 1) is zero and the quantity produced by the rival is positive. The pink region represents combinations of prices that will never be selected by firms as the profit, at least for one player, will be too low or even negative when prices are set below (resp. to the left) of the straight line $p_{2}=w\left(\right.$ resp. $\left.p_{1}=w\right)$.

Gradient dynamics are economically well-defined only for trajectories that belong to $A \cap B$ for any $n$, that is when both demands and profits are strictly positive. If the gradient dynamics defines a trajectory for which the couple of prices $\left(p_{1}, p_{2}\right)$ at a certain iterate belongs to one of the two subsets (the yellow region or the green region in Figure 1) whose union defines set $A \backslash B$, then one of the two firms does not produce because the demand of consumers is zero (i.e., the price is fixed at too high a level in that case) and its profits are null. As the profit function of that firm is null on the subset of $A \backslash B$ considered, the related marginal profit is zero. Then, by following the mechanism of gradient dynamics the firm will continue to choose the same 
price for the next period. However, from an economic point of view, it seems more reasonable to assume that the firm facing a zero-demand modifies its choices and then reduces the price to make it more likely facing a positive demand and therefore positive profits. Specifically, we assume that if the price of firm $i$ is fixed at too high a level so that trajectories exit $A \cap B$, then production is zero and firm $i$ will decide to reduce its price to get positive profits. This rule can be summarised by the dynamic equation $p_{i}^{\prime}=p_{i}-\beta\left(p_{i}-\bar{w}\right)$, where $\beta \in(0,1) .{ }^{5}$ Now, define

$$
\begin{aligned}
& H\left(p_{1}, p_{2}\right):=p_{1}+\alpha \frac{p_{1}^{1-\eta}(1-\eta)+p_{1}^{-\eta} \eta w-d p_{1} p_{2}^{-\eta}}{1-d^{2}} \\
& K\left(p_{1}, p_{2}\right):=p_{2}+\alpha \frac{p_{2}^{1-\eta}(1-\eta)+p_{2}^{-\eta} \eta w-d p_{2} p_{1}^{-\eta}}{1-d^{2}}
\end{aligned}
$$

as the equations that characterise the gradient dynamics. Then, by assuming that firms unilaterally set the price and dynamically prefer a trajectory leading to positive profits rather than zero profits $\left(p_{i}^{\prime} \geq \bar{w}\right)$, then map $T: A \rightarrow A$ that governs the dynamics of the system depending on whether the non-negativity constraints are binding or not is specified in the following way (for a pictorial view of how map $T$ acts see Figure 2):

$$
T_{A}:\left\{\begin{array}{l}
p_{1}^{\prime}=H\left(p_{1}, p_{2}\right) \\
p_{2}^{\prime}=K\left(p_{1}, p_{2}\right)
\end{array},\right.
$$

if $\bar{w}<p_{1}<d^{\frac{-1}{\eta}} p_{2}, \bar{w}<p_{2}<d^{\frac{-1}{\eta}} p_{1}, H\left(p_{1}, p_{2}\right) \geq \bar{w}$ and $K\left(p_{1}, p_{2}\right) \geq \bar{w}$;

$$
T_{B}:\left\{\begin{array}{c}
p_{1}^{\prime}=p_{1}-\beta\left(p_{1}-\bar{w}\right) \\
p_{2}^{\prime}=K\left(p_{1}, p_{2}\right)
\end{array}\right.
$$

if $p_{1} \geq d^{\frac{-1}{\eta}} p_{2}, \bar{w}<p_{2}<d^{\frac{-1}{\eta}} p_{1}$ and $K\left(p_{1}, p_{2}\right) \geq \bar{w}$;

$$
T_{C}:\left\{\begin{array}{c}
p_{1}^{\prime}=H\left(p_{1}, p_{2}\right) \\
p_{2}^{\prime}=p_{2}-\beta\left(p_{2}-\bar{w}\right)
\end{array},\right.
$$

if $\bar{w}<p_{1}<d^{\frac{-1}{\eta}} p_{2}, p_{2} \geq d^{\frac{-1}{\eta}} p_{1}$ and $H\left(p_{1}, p_{2}\right) \geq \bar{w}$;

$$
T_{D}:\left\{\begin{array}{c}
p_{1}^{\prime}=\bar{w} \\
p_{2}^{\prime}=p_{2}-\beta\left(p_{2}-\bar{w}\right)
\end{array},\right.
$$

if $p_{2} \geq d^{\frac{-1}{\eta}} p_{1}$ and $H\left(p_{1}, p_{2}\right)<\bar{w}$;

$$
T_{E}:\left\{\begin{array}{c}
p_{1}^{\prime}=p_{1}-\beta\left(p_{1}-\bar{w}\right) \\
p_{2}^{\prime}=\bar{w}
\end{array},\right.
$$

if $p_{1} \geq d^{\frac{-1}{\eta}} p_{2}$ and $K\left(p_{1}, p_{2}\right)<\bar{w}$;

\footnotetext{
${ }^{5}$ We note that the price is always larger than $\bar{w}$.
} 


$$
T_{F}:\left\{\begin{array}{c}
p_{1}^{\prime}=\bar{w} \\
p_{2}^{\prime}=K\left(p_{1}, p_{2}\right)
\end{array},\right.
$$

if $\bar{w}<p_{2}<d^{\frac{-1}{\eta}} p_{1}, K\left(p_{1}, p_{2}\right) \geq \bar{w}$ and $H\left(p_{1}, p_{2}\right)<\bar{w}$;

$$
T_{G}:\left\{\begin{array}{c}
p_{1}^{\prime}=H\left(p_{1}, p_{2}\right) \\
p_{2}^{\prime}=\bar{w}
\end{array},\right.
$$

if $\bar{w}<p_{1}<d^{\frac{-1}{\eta}} p_{2}, H\left(p_{1}, p_{2}\right) \geq \bar{w}$ and $K\left(p_{1}, p_{2}\right)<\bar{w}$

$$
T_{H}:\left\{\begin{array}{l}
p_{1}^{\prime}=\bar{w} \\
p_{2}^{\prime}=\bar{w}
\end{array},\right.
$$

if $H\left(p_{1}, p_{2}\right)<\bar{w}$ and $K\left(p_{1}, p_{2}\right)<\bar{w}$.

In general map $T$ is not continuous or not smooth along boundaries that separate the different regions of its definition. In particular, along the half-lines $p_{i}=\bar{w}, p_{-i}>\bar{w}$ the map is continuous but not smooth, whereas along the half-lines $p_{i}=d^{\frac{-1}{\eta}} p_{-i}, p_{-i}>\bar{w}$ the map is non-continuous. In addition, map $T$ is non-invertible. This implies that given a point belonging to set $F$, several preimages can exist. As map $T$ is defined by eight different pieces, it is not easy to classify the number of preimages associated to the regions of set $F$. Thus, in order to study the absorbing areas, that is the regions bounded by critical curves of finite rank $L C_{n}$, with $n=0 \ldots l$, we resort to numerical simulations. In what follows, we will see that in some cases the critical curves involved in defining absorbing regions will be only those defined by points such that the determinant of map $T_{A}$ is null. However, in other cases the lines that define the boundaries in $F$ of regions in which the single maps $T_{i}(i=A, \ldots, H)$ hold may also be involved.

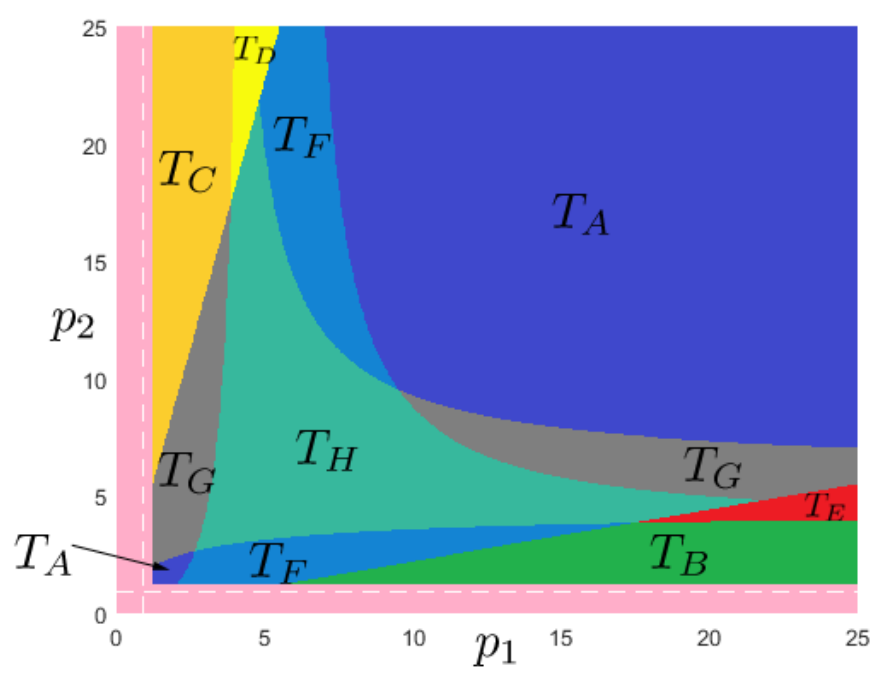


Figure 2. Regions of phase plane $\left(p_{1}, p_{2}\right)$ bounded by map $T_{i}(i=A, \ldots, H)$ are evidenced by different colours. The dark-blue region is described by map $T_{A}$. The green region is described by map $T_{B}$. The orange region is described by map $T_{C}$. The yellow region is described by map $T_{D}$. The red region is described by map $T_{E}$. The light-blue region is described by map $T_{F}$. The grey region is described by map $T_{G}$. The aqua green region is described by map $T_{H}$. The pink region represents combinations of prices that will never be selected by firms as the profit, at least for one player, will be too low or even negative when prices are set below (resp. to the left) of the straight line $p_{2}=w\left(\right.$ resp. $\left.p_{1}=w\right)$.

Map $T$ can produce only attractors at finite distance due to the presence of constraints. Consider a sequence $\left(p_{1}, p_{2}\right)$ with $\left\|p_{1}, p_{2}\right\| \rightarrow+\infty$. If $p_{i}$ takes a finite value then the sequence will enter $A \backslash B$ region from which $p_{-i}^{\prime}=p_{-i}-\beta\left(p_{-i}-\bar{w}\right)<p_{-i}, i=1,2$. Instead, if both components of the sequence positively diverge, we have that $H\left(p_{1}, p_{2}\right)<\bar{w}$ for $p_{2}>\frac{\alpha d}{1-d^{2}}$ and $K\left(p_{1}, p_{2}\right)<\bar{w}$ for $p_{1}>\frac{\alpha d}{1-d^{2}}$. From this, it follows that there exists an iterate such that $\left(p_{1}, p_{2}\right)=(\bar{w}, \bar{w})$. It is now sufficient to show that there exists a maximum value taken by $p_{i}^{\prime}$ when map $T_{A}$ works. By referring for simplicity to $p_{1}^{\prime}$ (for $p_{2}^{\prime}$ the line of reasoning is analogous), $H\left(p_{1}, p_{2}\right)$ is defined on set

$$
\Lambda_{1}=\left\{\left(p_{1}, p_{2}\right) \in R^{2}: p_{1} \geq \bar{w}, p_{2} \geq \bar{w}, p_{2} \geq p_{1} d^{1 / \eta}\right\}
$$

As there exist feasible points in $\Lambda_{1}$ for which $p_{1}^{\prime}=\bar{w}$, the solutions of the problem $\max _{\left(p_{1}, p_{2}\right) \in \Lambda_{1}} H\left(p_{1}, p_{2}\right)$ coincide with the solutions of the problem $\max _{\left(p_{1}, p_{2}\right) \in \Lambda_{2}} H\left(p_{1}, p_{2}\right)$, where

$$
\Lambda_{2}=\left\{\left(p_{1}, p_{2}\right) \in R^{2}: p_{1} \geq \bar{w}, p_{2} \geq \bar{w}, p_{2} \geq p_{1} d^{1 / \eta}, H\left(p_{1}, p_{2}\right) \geq \bar{w}\right\}
$$

It is easy to verify that $\Lambda_{2}$ is a compact set and $H\left(p_{1}, p_{2}\right)$ is a continuous function. By applying the Extreme Value Theorem, we get the result. In particular, the solution will be located on constraints $p_{2}=\bar{w}$ or $p_{2}=p_{1} d^{1 / \eta}$ depending on the value of parameters.

Map $T$ defined in (29) has a unique fixed point (that coincides with Nash equilibrium 15), whose coordinate value are $E^{*}=\left(p_{1}^{*}, p_{2}^{*}\right)=\left(\frac{\eta w}{d+\eta-1}, \frac{\eta w}{d+\eta-1}\right)$. Map $T$ is symmetric, i.e. it does not change if $p_{1}$ and $p_{2}$ are swapped, that is $T \circ W=W \circ T$, where $W:\left(p_{1}, p_{2}\right) \rightarrow\left(p_{2}, p_{1}\right)$. This implies that the diagonal $\Delta=\left\{\left(p_{1}, p_{2}\right): p_{1}=p_{2}\right\}$ is an invariant manifold, i.e. the dynamics lie on $\Delta$ for every $t$ by starting from $p_{1}(0)=p_{2}(0)$. In this case, the behaviour of the dynamic system is described by the restriction of $T$ on $\Delta$, and synchronised trajectories (i.e., $p_{1}(t)=p_{2}(t)$ for every $t$ ) are governed by map $T_{\Delta}: \Delta \rightarrow \Delta$ defined as follows:

$$
T_{\Delta}: p^{\prime}=f(p):=\max \{\bar{w}, g(p)\}
$$

where $g(p):=p+\frac{\alpha[p(1-\eta-d)+\eta w]}{p^{\eta}\left(1-d^{2}\right)}$. We note that $g(p)$ defines a unimodal function, first decreasing and then increasing. Let $p_{\min }$ be the minimum of such an expression. If $g\left(p_{\min }\right) \geq \bar{w}$ then the dynamics of the map at every iterate will be governed by $g(p)$. Instead, if we have $g\left(p_{\min }\right)<\bar{w}$ 
then there exist two points $\underline{p}$ and $\bar{p}$, with $\underline{p}<\bar{p}$, such that $f(p)$ can be rewritten in the following way:

$$
f(p)=\left\{\begin{array}{c}
p+\frac{\alpha[p(1-\eta-d)+\eta w]}{p^{\eta}\left(1-d^{2}\right)}, \quad \text { if } \quad p<\underline{p} \quad \text { or } \quad p>\bar{p} \\
\bar{w}, \quad \text { if } \quad \underline{p} \leq p \leq \bar{p}
\end{array}\right.
$$

The following proposition defines the local stability conditions of the fixed point of map $T_{\Delta}$.

Lemma 5 Map $g(p)$ always admits a fixed point $p^{*}=\frac{\eta w}{d+\eta-1}$.

Proposition 6 If $\bar{w}<p^{*}$ then $p^{*}$ is a fixed point of $f(p)$ and it is locally asymptotically stable for $\alpha<\alpha_{T H}$ and unstable for $\alpha>\alpha_{T H}$, where

$$
\alpha_{T H}:=\frac{2 \eta w\left(1-d^{2}\right)}{\eta w\left(\eta^{2}-\eta+d\right)\left(\frac{\eta w}{d+\eta-1}\right)^{-\eta}-(1-\eta)^{2}(d+\eta-1)\left(\frac{\eta w}{d+\eta-1}\right)^{1-\eta}} .
$$

If $\bar{w}>p^{*}$ then $\bar{w}$ is a fixed point of $f(p)$ and it is locally super stable.

If $g\left(p_{\min }\right) \geq \bar{w}$ is true for every $\alpha$ then we have the classical process of period doubling when $\alpha$ increases $(f(p)=g(p))$. Instead, when $\alpha$ varies map $g(p)$ collides with constraint $\bar{w}$ (Figure $3(\mathrm{a}))$. Then, the period doubling process undergoes a change, as shown in Figure 3(b). In particular, for $\alpha>30.1$ the two-piece chaotic attractor is replaced with a $n$-period cycle (where $n$ depends on the value of $\alpha$ ) and the points of the cycle are given by $\bar{w}$ and its subsequent $n-1$ iterates (see Sushko et al., 2014 for an in-depth analysis on the role of a flat branch in the definition of the map).

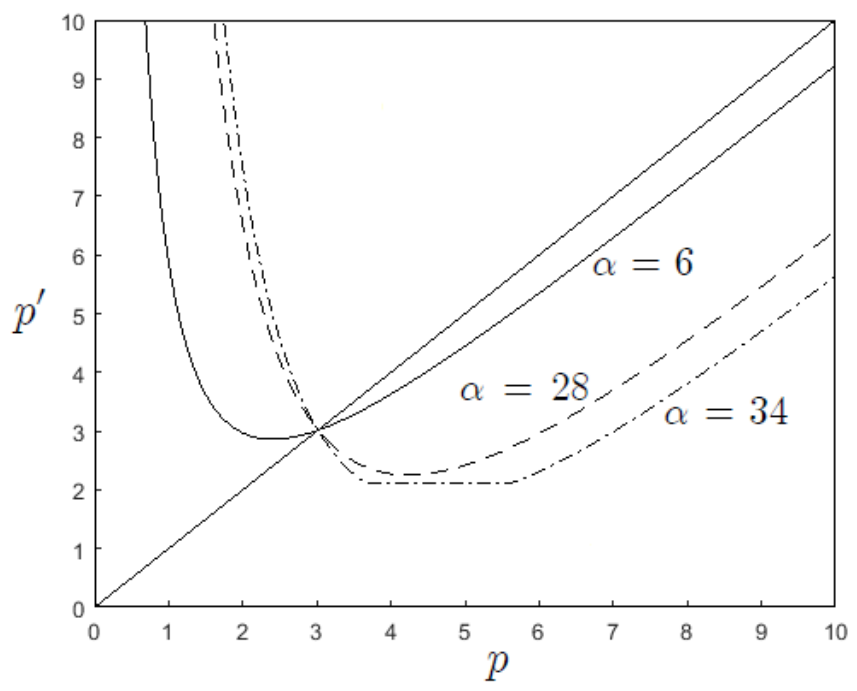

(a) 


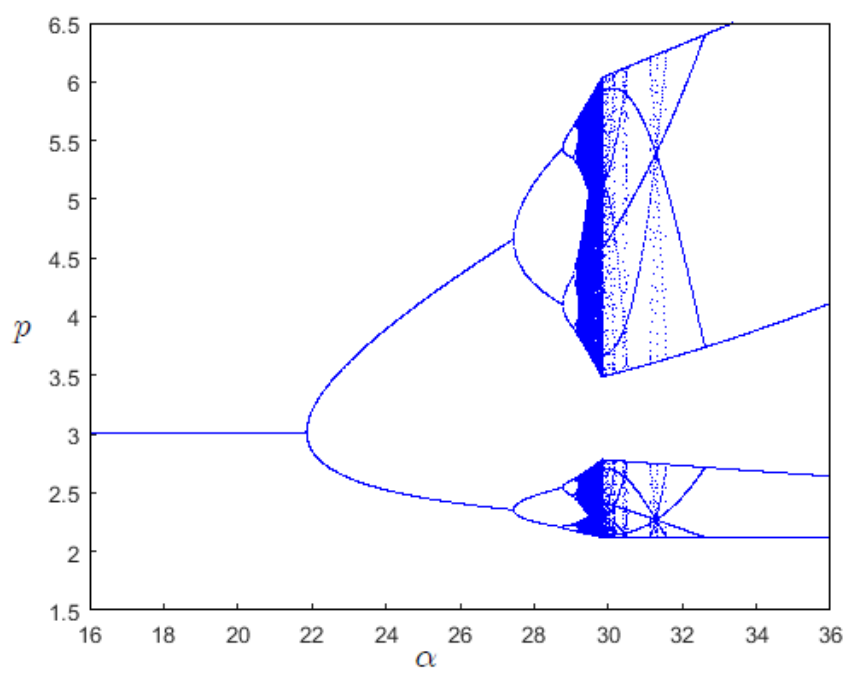

(b)

Figure 3. Parameter set: $d=0.06, \eta=1.34, w=0.9$ and $\bar{w}=2.12$. (a) When $\alpha=6$ (solid line), the stationary equilibrium of map for $T_{\Delta}$ is locally asymptotically stable. When $\alpha=28$ (dashed line), the stationary equilibrium of map for $T_{\Delta}$ is unstable (in this case there exists a four-period cycle, as shown in the bifurcation diagram, Figure 3(b)) When $\alpha=34$ (dot-dashed line), we note the change undergone by the map as the constraint $\bar{w}$ is binding (see also the bifurcation diagram). (b) Bifurcation diagram for $\alpha$.

By using numerical simulations, in what follows we show some dynamic (global) phenomena produced by map $T$ when the constraints on quantities and profits are not binding. Therefore, the dynamics of the model are described by (29), according to which prices change over time by following a Bischi-like gradient adjustment mechanism. In the exercises summarised in Figures 4-12 we use the parameter set: $d=0.01, \alpha=10, \beta=0.4, w=0.92, \varepsilon=0.14$ and $\bar{w}=w+\varepsilon$, and let $\eta$ vary.

Let us begin the simulation exercise by considering $\eta=1.5$, a situation for which the unique attractor of map $T$ is the Nash equilibrium of the game, $E^{*}$ (Figure 4). An increase in $\eta$ causes a transverse flip bifurcation (this holds at $\eta \cong 1.715$ ), which in turn gives birth to a two-period cycle out of the diagonal. This configuration of the phase plane is depicted in Figure 5 showing for $\eta=1.745$ that the unique attractor of the system is a two-period cycle out of the diagonal ( $R_{1}$ and $R_{2}$ in the figure). However, an investigation along the diagonal shows the existence of a two-period cycle attracting for the map restricted on the diagonal $\left(T_{\Delta}\right)$, whose points are labelled as $V_{1}$ and $V_{2}$ (not reported in Figure 5), but transversely unstable and then unstable for map $T$. When $\eta$ is around 1.75, this last two-period cycle becomes also transversely stable, as depicted in Figure 6. The figure shows the phase plane for $\eta=1.8$, where a stable two-period cycle on the diagonal (red points $V_{1}$ and $V_{2}$ ), whose basin of attraction is light-grey coloured, coexists with a two-period cycle out of the diagonal (black points $R_{1}$ and $R_{2}$ ), whose basin of attraction is dark-grey coloured. Around $\eta=1.841$ the two-period cycle out of the diagonal 
undergoes a Neimark-Sacker bifurcation. To this purpose, Figure 7 (plotted for $\eta=1.85$ ) shows the coexistence of two invariant closed curves (depicted in black and labelled $\Gamma_{1}$ and $\Gamma_{2}$ ) out of the diagonal, attracting all trajectories that belong to the dark-grey region, and a two-period cycle on the diagonal (red points $V_{1}$ and $V_{2}$ ), attracting all trajectories that belong to the light-grey region. When $\eta$ increases further, a saddle node bifurcation gives birth to an additional attractor. Indeed, Figure 8 displays the coexistence of three attractors for $\eta=1.87$. In particular, we observe a two-period cycle along the diagonal (red points $V_{1}$ and $V_{2}$ ), whose basin of attraction is depicted in yellow, and a couple of eight-period cycles, black points and white points in the figure, whose basins of attraction are light-grey coloured and dark-grey coloured, respectively. A further slight reduction in $\eta(\eta=1.88)$ causes the appearance of a couple of four-piece chaotic attractors (the black one is $\Psi_{11}, \Psi_{12}, \Psi_{13}$ and $\Psi_{14}$, and the blue is $\Psi_{21}, \Psi_{22}, \Psi_{23}$ and $\Psi_{24}$, which are not reported in the figure to avoid confusion) and a four-period cycle on the diagonal (red points $V_{1}, V_{2}, V_{3}$ and $V_{4}$ ), see Figure 9 . Interestingly, when $\eta=1.885$ the two four-piece chaotic attractors has become a unique two-piece chaotic attractor (depicted in black and labelled $\Psi_{1}$ and $\Psi_{2}$ in Figure 10), which continues to coexist with a four-period cycle on the diagonal (red points $V_{1}, V_{2}, V_{3}$ and $V_{4}$ ). Finally, for $\eta=2$ the two-piece chaotic attractor has become a unique chaotic attractor $(\Psi)$, as shown in Figure 11.

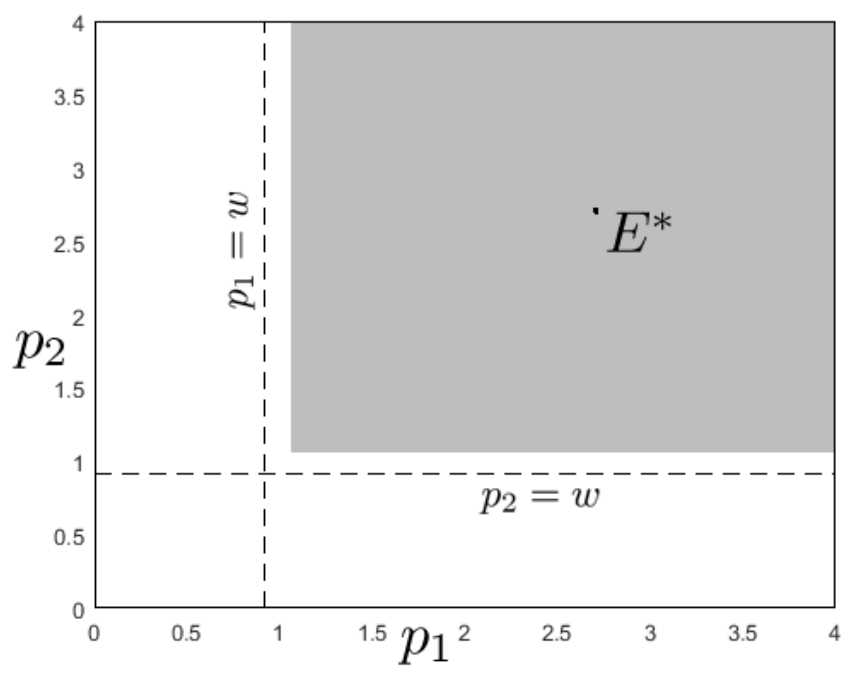

Figure 4. Basin of attraction of the stable fixed point $E^{*}$ (a one-period cycle on the diagonal) of map $T$ for $\eta=1.5$. 


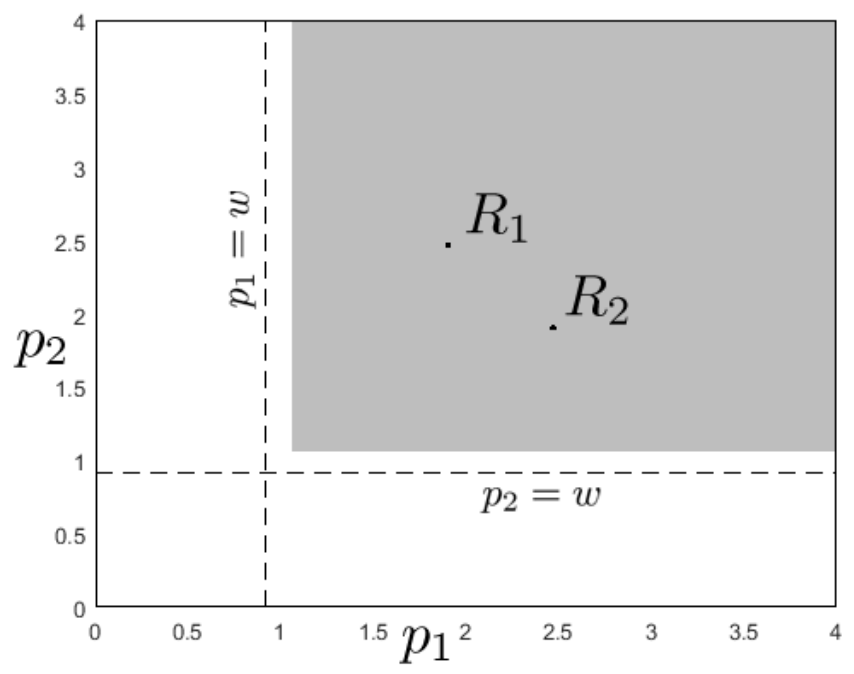

Figure 5. A transverse flip bifurcation produced a two-period cycle (unstable diagonal), $R_{1}$ and $R_{2}$. There exists no attracting cycle along the diagonal $(\eta=1.745)$.

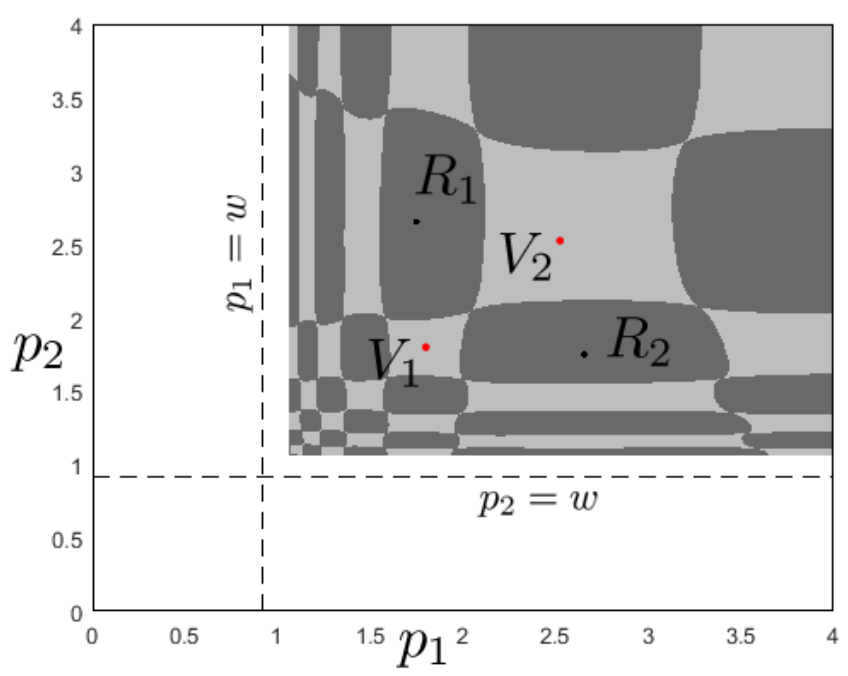

Figure 6. A stable two-period cycle on the diagonal (red points $V_{1}$ and $V_{2}$ ), whose basin of attraction is light-grey coloured, coexists with a two-period cycle out of the diagonal (black points $R_{1}$ and $R_{2}$ ), whose basin of attraction is dark-grey coloured $(\eta=1.8)$. 


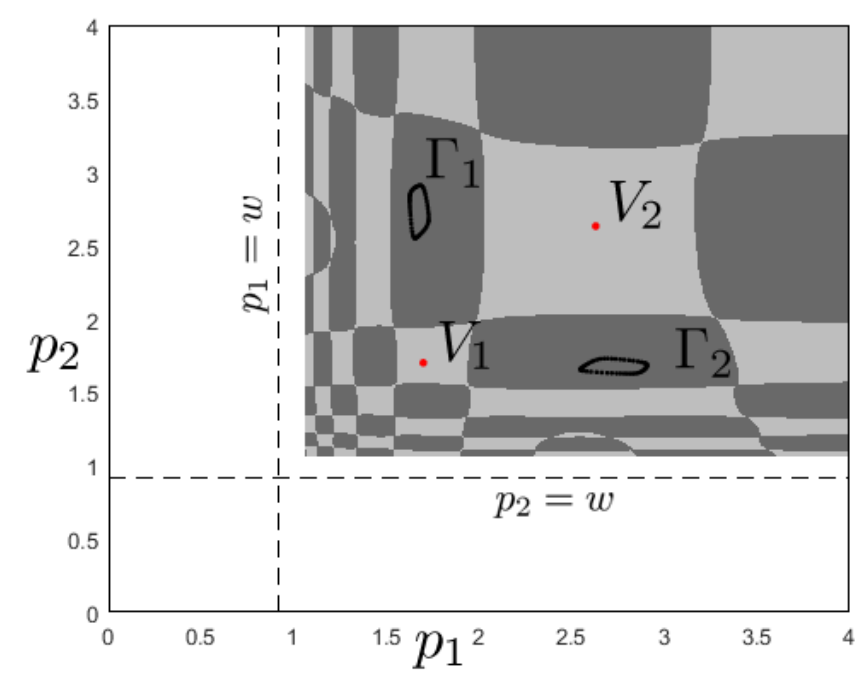

Figure 7. Coexistence of two invariant closed curves $\Gamma_{1}$ and $\Gamma_{2}$ (depicted in black) out of the diagonal, born through a Neimark-Sacker bifurcation, that attract all trajectories that belong to the dark-grey region, and a two-period cycle on the diagonal (red points $V_{1}$ and $V_{2}$ ), that attracts all trajectories that belong to the light-grey region $(\eta=1.85)$.

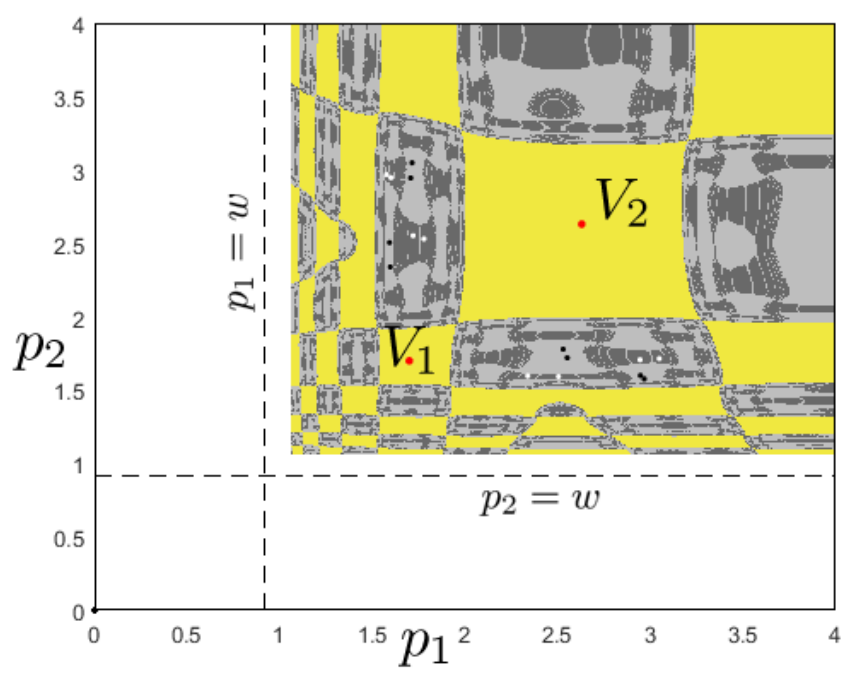

Figure 8. A saddle node bifurcation gave birth to a third attractor. The figure shows the coexistence of a two-period cycle along the diagonal (red points $V_{1}$ and $V_{2}$ ), whose basin of attraction is depicted in yellow, and a couple of eight-period cycles, black points and white points, whose basins of attraction are light-grey coloured and dark-grey coloured, respectively $(\eta=1.87)$. 


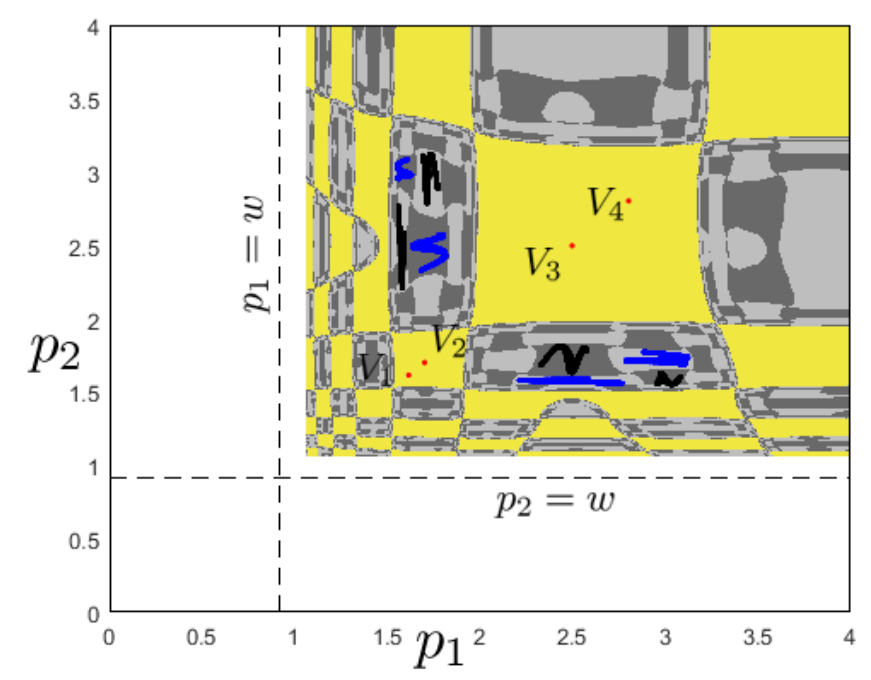

Figure 9. A couple of four-piece chaotic attractors coexists with a four-period cycle on the diagonal. The black chaotic attractor is denoted by $\Psi_{11}, \Psi_{12}, \Psi_{13}$ and $\Psi_{14}$ (this was done in accordance with the left-to-right reading direction), whereas the blue one is denoted with $\Psi_{21}$, $\Psi_{22}, \Psi_{23}$ and $\Psi_{24}$. The four-period cycle on the diagonal is denoted by red points $V_{1}, V_{2}, V_{3}$ and $V_{4}(\eta=1.88)$.

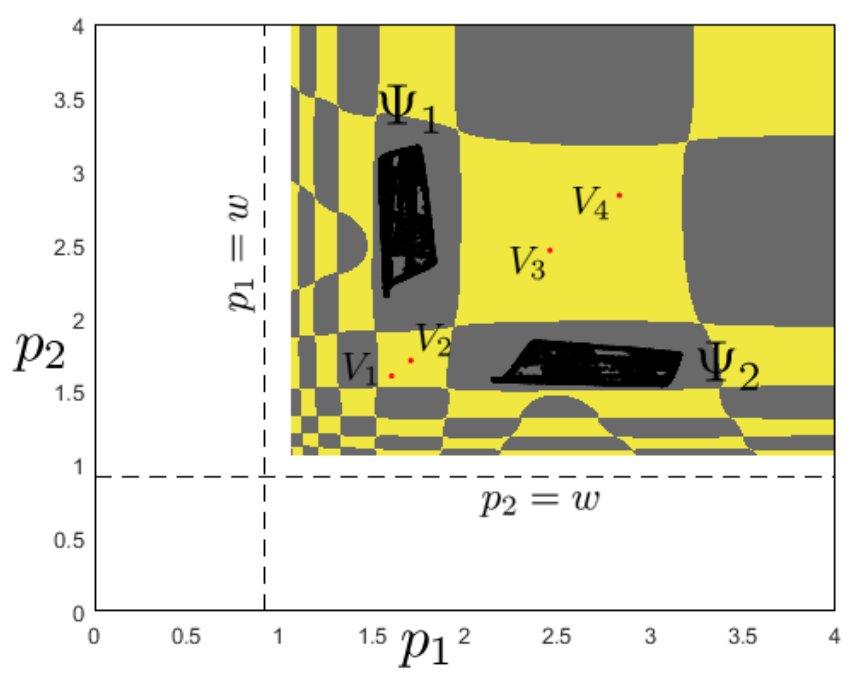

Figure 10. A two-piece chaotic attractor, depicted in black and denoted by $\Psi_{1}$ and $\Psi_{2}$, coexists with a four-period cycle on the diagonal, depicted in red and denoted by $V_{1}, V_{2}, V_{3}$ and $V_{4},(\eta=1.885)$. 


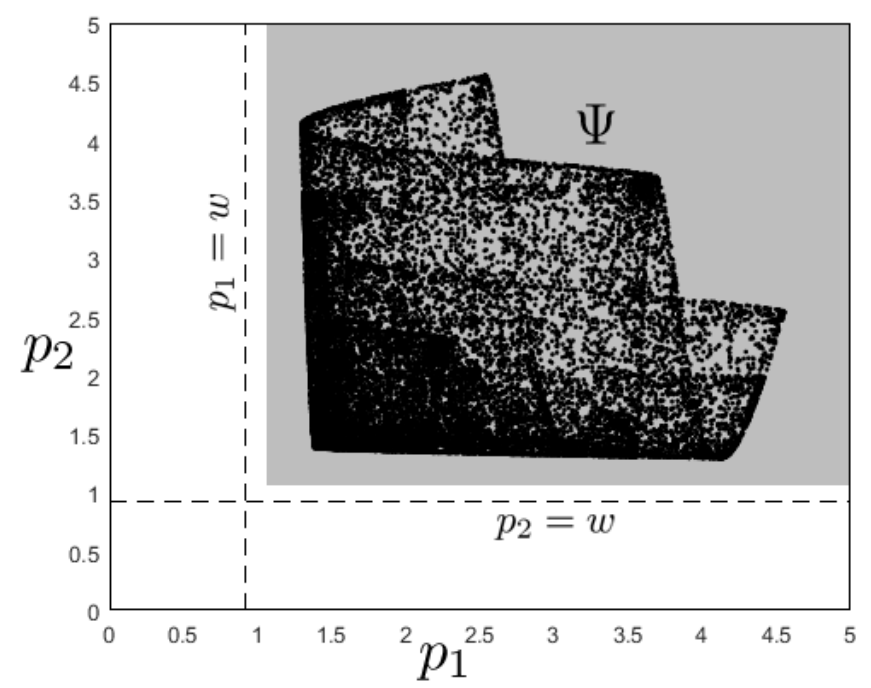

Figure 11. A unique chaotic attractor $\Psi(\eta=2)$.

As explained above, the attractors related to all numerical exercises from Figure 4 to Figure 11 are generated by using map (29), whose dynamics are described by a Bischi-like gradient mechanism. However, the other maps - from (30) to (36) - have been used to define the basins of attraction. In fact, the relative simplicity of the basins of attraction obtained when only one attractor for the system exists is closely related to the presence of economic constraints and different adjustment mechanisms. If these constraints were not used, and then we considered the hypothesis that the model was described only by (29) for each pair of positive prices (in which case we would have values of prices corresponding to negative revenue per unit of product and/or negative quantities for some iterates), then there would exist some sets of initial conditions whose corresponding forward dynamics would not be well-defined. This means that there would exist an iterate such that the trajectory should go in the region of negativity of prices, thus making impossible the calculation of the subsequent iterates due to the existence of an exponent term between 0 and 1 of prices. As an example, in Figure 12 we depict the basin of attraction generated by assuming that the dynamics of the model are described only by (29) for any $\left(p_{1}, p_{2}\right) \in R_{++}^{2}$ (see Fanti et al., 2013a for a study of the shape of the boundary of the basin of attraction in a Bertrand model with linear demand but without constraints). 


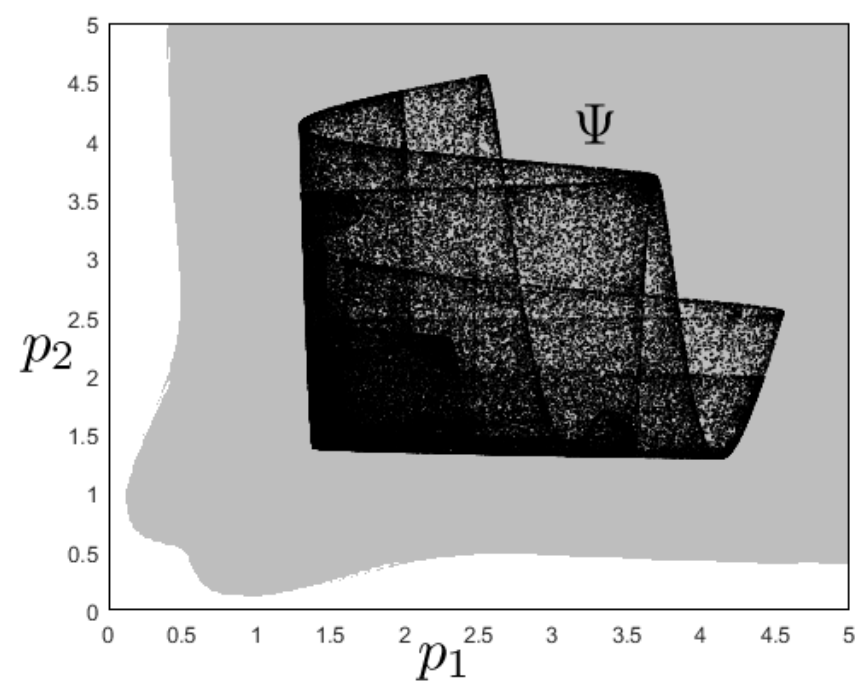

Figure 12. Attractor and basin of attraction when no constraints are introduced for the same parameter values as in Figure 11.

\section{The role of constraints}

In this section, we analyse the role of economic constraints on the dynamics of a nonlinear Bertrand duopoly, where the price is the strategic variable.

Non-negativity of profits. In order to make the reading of the rest article as clear as possible, we consider the following parameter set: $d=0.014, \alpha=33.9, \beta=0.01, \eta=1.4, w=0.9$, $\bar{w}=w+\varepsilon$ and let $\varepsilon$ vary (an increase in $\varepsilon$ captures a rise in the average and marginal cost of production). For $\varepsilon=0.05$, a unique $\omega$-limit set exists for the system, as is shown in Figure 13(a). We recall that as map $T$ is non-differentiable and/or discontinuous along some lines, the critical curves of rank-1 may be given by 1) the locus of points which are images of the set where the Jacobian determinant vanishes $T(\operatorname{det}(J)=0)$, where the set defined by $\operatorname{det}(J)=0$ is $\operatorname{denoted}$ as $L C_{-1}$, set of merging preimages, 2 ) the points where map $T$ results to be non-differentiable, 3) the points where the map is not continuous. Critical curves of higher rank are given by the forward iterates of rank-1 curves. With the parameter set used in the numerical simulations, the attractor completely lies in the region of the phase plane where map $T_{A}$ acts. Then, we restrict the analysis to the study of properties of map $T_{A}$. Irrespective of the domain of definition, $T_{A}$ is in general non-invertible and its $L C_{-1}$ curves are a subset of points corresponding to which the determinant of $T_{A}$ is zero, that is $L C_{-1}\left(T_{A}\right) \subseteq\left\{\left(p_{1}, p_{2}\right) \in R_{+}^{2}: \operatorname{det}\left(J\left(T_{A}\right)\right)=0\right\}$. Unfortunately, in this case the expression of the determinant of the Jacobian matrix does not define a classical geometrical curve. However, through numerical simulations we are able to verify that such a curve is made up of two branches, $L C_{-1}^{a}$ and $L C_{-1}^{b}$. Figure $13(\mathrm{~b})$ shows the portion of $L C_{-1}^{a}$ (bold red line) involved in the definition of the absorbing region as well as its 
first three iterates (red lines). An increase in $\varepsilon$ implies that lines $p_{i}=\bar{w}(i=1,2)$ become closer and closer to the chaotic attractor. A border collision occurs at $\varepsilon \cong 0.283$ and Figures 14 (a) and 14(b) show the role played by the constraint on the marginal cost of production (related to the non-negativity of profits) in the definition of the shape of the attractor when $\varepsilon=0.4$. In this case, the dynamics of the attractor are defined by maps $T_{A}, T_{F}$ and $T_{G}$ (the quantity produced by both firms and demanded in the market are always positive). The existence of a binding constraint (that prevents to set the prices below the marginal cost) has restricted the area of the chaotic attractor. The dashed (resp. solid) portion of the lines in Figures 13(b) and 14(b) describes the non-binding (resp. binding) part of the constraints. The constraints are depicted in blue in both figures. The same graphical style is used with regard to both the constraints on the non-negativity of unitary profits (the vertical and horizontal lines) and the constraints on the quantities produced by firm 1 and firm 2 and demanded on the market (the two lines with positive slope). The action of this kind of constraints has a natural effect from an economic point of view, in the sense that it prevents firms from setting too high prices leading to low or null demands and profits.

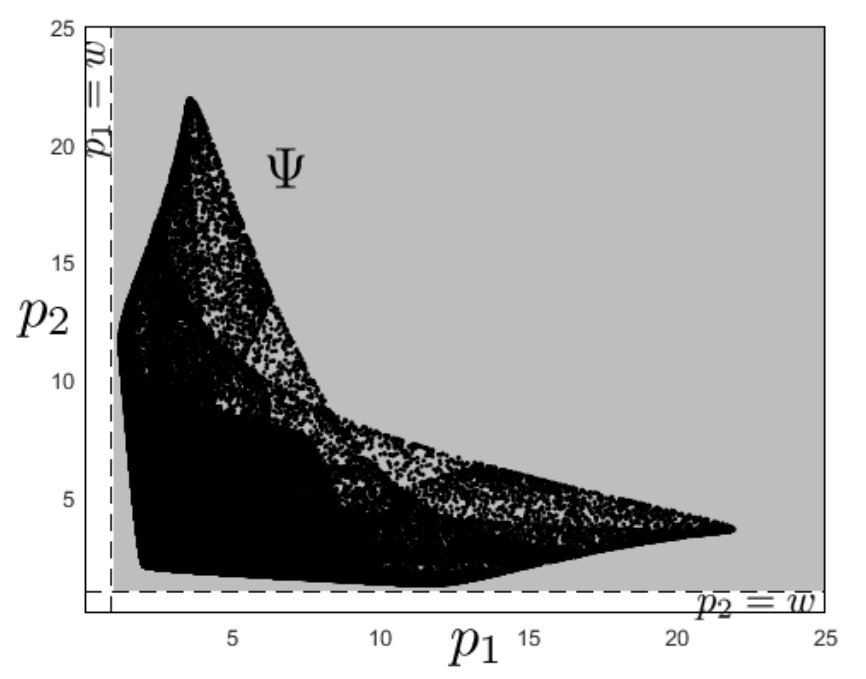

(a) 


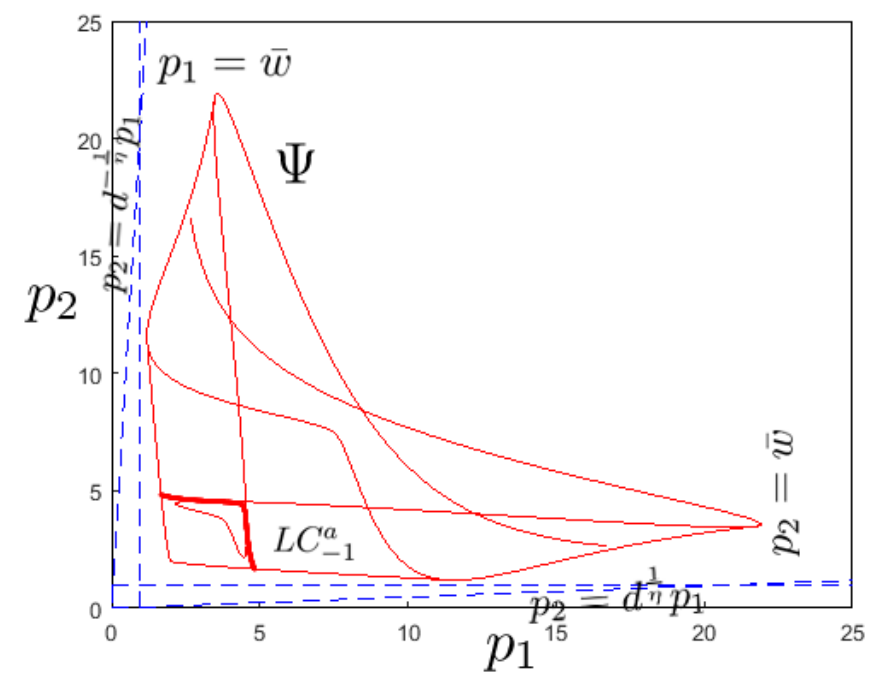

(b)

Figure 13. (a) A unique chaotic attractor $\Psi$ captures all the dynamics of the system. On the attractor, quantities of both firms are positive and prices are larger than (the constraint) $\bar{w}$. (b) Critical curves generated by $L C_{-1}$ and their subsequent iterates define the chaotic area found by simulations in Figure 13(a).

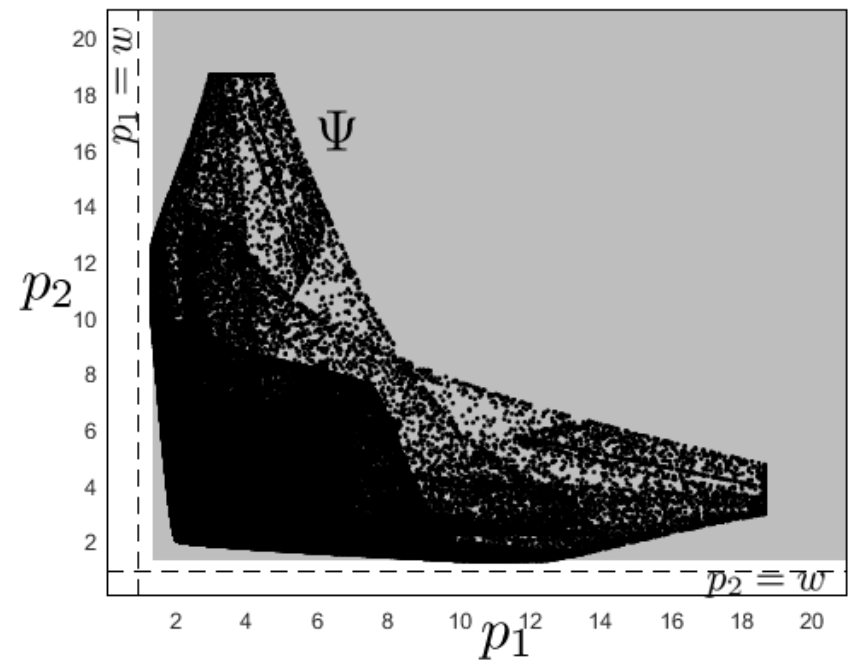

(a) 


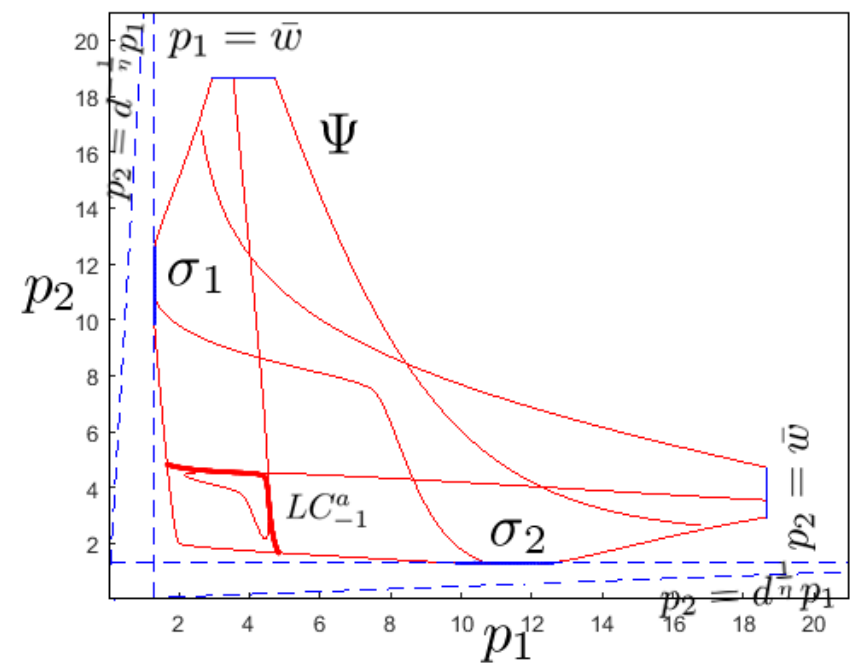

(b)

Figure 14. (a) A unique chaotic attractor $\Psi$ captures all the dynamics of the system. On the attractor, both quantities are positive but the non-negativity constraints on profits, i.e. the vertical and horizontal dashed lines, are binding. (b) Critical curves generated by $L_{-1}$ and by portions of the straight lines $p_{i}=\bar{w}(i=1,2)$.

Non-negativity of quantities. This part of the section is devoted to describe the role of the constraints related to the non-negativity of quantities (the two lines with positive slope) on longterm dynamics. We will see that when a border collision bifurcation occurs, these constraints substantially modify the dynamics of the system. Specifically, they change the shape as well as the type of the $\omega$-limit set. This result accords with the analysis of Tramontana et al. (2010), in which a Cournot model with constraints on the reaction curves of the firms is studied. By starting from the parameter set used to plot Figures 14(a) and 14(b) and increasing $d$ we have a border collision when $d \cong 0.02402$. This implies that the attractor collides with the nonnegativity constraints on quantities. By letting $d$ increase further $(d=0.031)$, we have that portions of the attractor lie in $A \backslash B$ region. Due to the reinjection mechanism introduced in Section 3 for maps $T_{B}, T_{C}, T_{D}$ and $T_{E}$, there is the birth of new portions of the attractor also in $A \cap B$ region. Indeed, the emergence of zero profits caused by zero demand for a firm generates (in this case) price strategies that were not possible when profits are positive at each iterate. This phenomenon is illustrated in Figures 15(a) and 15(b). However, economic constraints are responsible for other phenomena. In this regard, Figure 15(c) shows the coexistence of two symmetric attractors, each of which is made up by a ten-period cycle (this holds for $d=0.026)$. Nonetheless, the existence of a chaotic repeller significantly affects the convergence of trajectories towards such a $\omega$-limit set, as is shown in Figure 15(d). The existence of nonnegativity constraints and a reinjection mechanism induce a synchronisation of the trajectories along the diagonal when $d$ becomes larger. In this case, the symmetry is caused by the violation of the constraints on profits of both firms and then by coming into play the definition $T_{H}$ of the 
map. Instead, the subsequent iterate acts either on map $T_{A}$ or map $T_{H}$, so that the dynamics will be synchronised on the diagonal. This causes the birth of a global attractor given by a fourperiod cycle $(d=0.098)$. To this purpose, Figure $15(\mathrm{e})$ shows the time series of the difference between $p_{1}$ and $p_{2}$. It clearly reveals that after the 19 th iterate price dynamics are synchronised. Of course, when the other parameters of the model change, the dynamics along the diagonal can also be chaotic (to this purpose see the study of the dynamics along the diagonal in Section $3)$.

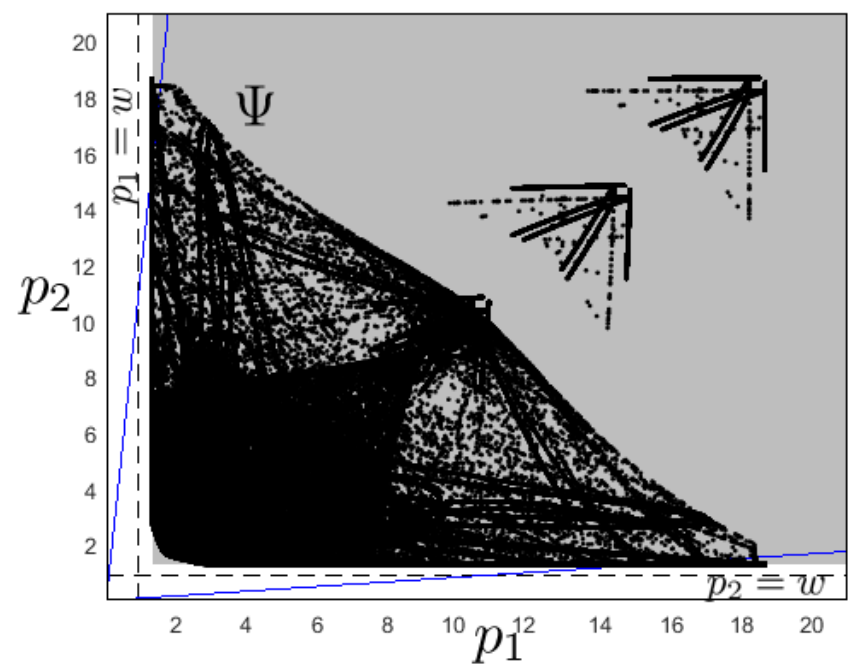

(a)

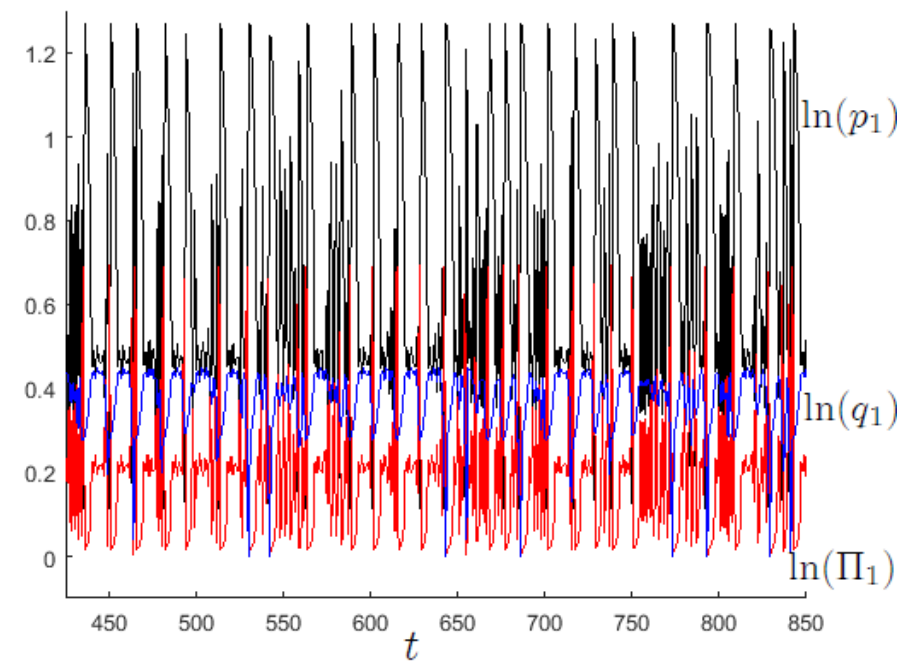

(b) 


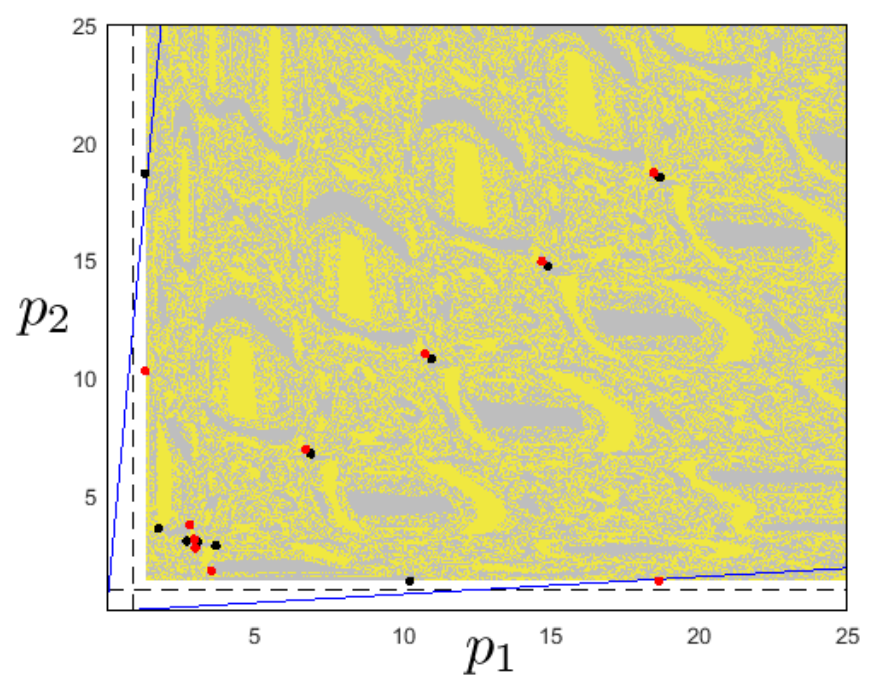

(c)

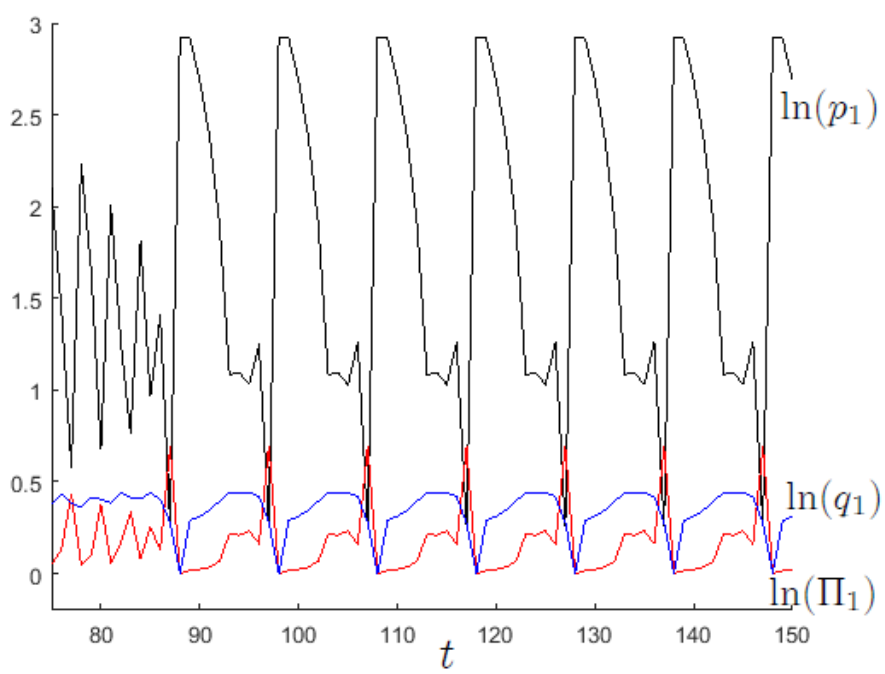

(d) 


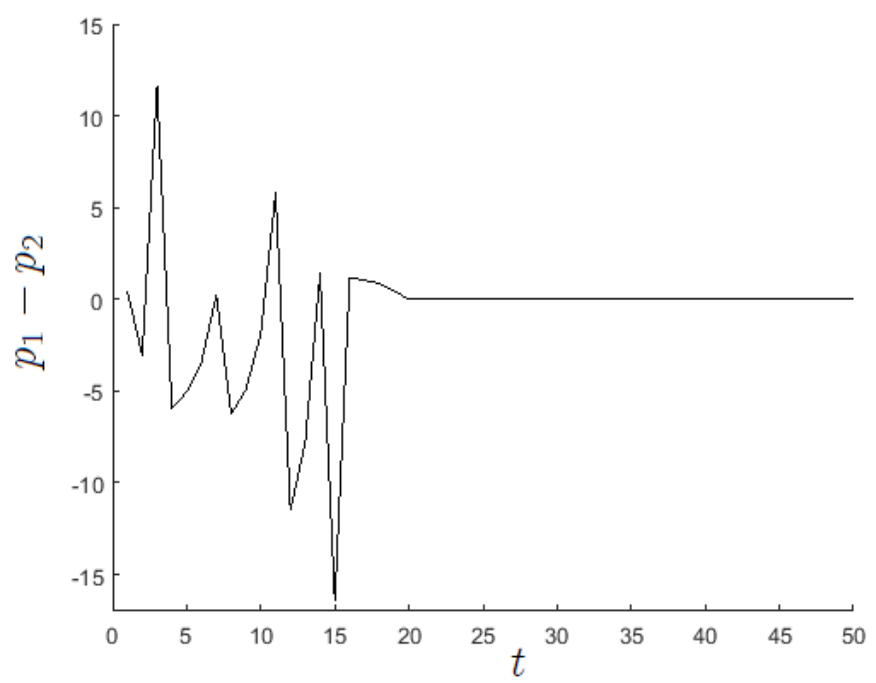

(e)

Figure 15. (a) A unique chaotic attractor $\Psi$ captures all the dynamics of the system $(d=0.031)$. Also in this case, the non-negativity constraints on profits are binding. Portions of the attractor lie in a region where the quantity produced by one of the two firms is zero. (b) Time series of the log of price (black), quantity (blue) and profit (red) of firm 1 associated to a typical trajectory of the system when $d=0.031$. We note that at some iterates of the time series $p_{1}$ and $p_{2}$ simultaneously take relatively high values corresponding to the two new portions of the attractor. By looking at Figure 15(b) one can note that when the price is set at too high a level, both demand and profits are zero. However, at the next iterate, consumers' demand and firms' profits turn to be positive due to a sharp reduction in price. (c) Basins of attraction (yellow and light-grey coloured) of two symmetric ten-period cycles (black and red points). Note that for any attractor there exists one point out of cone of the non-negativity constraints of quantities $(d=0.026)$. (d) Time series of the log of price (black), quantity (blue) and profit (red) of firm 1 associated to a typical trajectory of the system when $d=0.026$ (we may note the long transient). (e) Time series of the difference between $p_{1}$ and $p_{2}$ for $d=0.098$.

\section{Conclusions}

This article studied the dynamics of a nonlinear differentiated duopoly with price competition. The main aim is to emphasise the role of economic non-negativity constraints on long-term outcomes by assuming a Bischi-like gradient adjustment mechanism of prices (Bischi et al., 1998). The literature on nonlinear duopolies has been developed since the leading articles of Puu (1991) and Bischi et al. (1998), that analysed models with quantity competition with profit maximising firms and where players are assumed to have, respectively, complete information with static expectations and incomplete information with an adjustment mechanism based on the local estimate of their own marginal profits. 
The study of the Bertrand duopoly in a nonlinear setting has been scarcely tackled out, although an exception in this direction exists (Fanti et al., 2013a) that analysed dynamic price competition models with horizontal product differentiation and linear market demand and where profit-maximising firms have limited market knowledge. However, there are no contributions accounting for economic non-negativity constraints on profits and quantities. The existence of these constraints represents an important element that may substantially affect long-term dynamic outcomes. The present article wanted to fill this gap by considering a model with Bertrand rivalry. After an examination of the dynamics when prices are sufficiently large and quantities are positive, the article analysed the case in which at least one of these two conditions is violated. The existence of constraints can significantly affect the dynamics of the system and makes it possible observing new phenomena, such as synchronisation, which were not observable in the absence of them.

Acknowledgements The authors gratefully acknowledge Gian Italo Bischi, Davide Fiaschi, Laura Gardini, conference participants at NED 2013 held at University of Siena (Italy), NED 2015 held at Chuo University, Tokyo (Japan) and AMASES 2016 held at University of Catania (Italy) and seminar participants at University of Pisa. The authors also thank two anonymous reviewers for valuable comments and suggestions allowing an improvement in the quality of the work. The usual disclaimer applies.

Conflict of Interest: The authors declare that they have no conflict of interest.

\section{References}

[1] Agliari, A., Chiarella, C., Gardini, L., 2006. A re-evaluation of adaptive expectations in light of global nonlinear dynamic analysis. Journal of Economic Behavior \& Organization $60,526-552$.

[2] Agliari, A., Gardini, L., Puu, T., 2006. Global bifurcations in duopoly when the Cournot point is destabilized via a subcritical Neimark bifurcation. International Game Theory Review 8 1-20.

[3] Ahmed, E., Elsadany, A.A., Puu, T., 2015. On Bertrand duopoly game with differentiated goods. Applied Mathematics and Computation 251, 169-179.

[4] Andaluz, J., Jarne, G., 2016. Stability of vertically differentiated Cournot and Bertrandtype models when firms are boundedly rational. Annals of Operations Research 238, 1-25.

[5] Bischi, G.I., Lamantia, F., 2012. Routes to complexity induced by constraints in Cournot oligopoly games with linear reaction functions. Studies in Nonlinear Dynamics \& Econometrics 16 (Article 4), 1-28. 
[6] Bischi, G.I., Gallegati, M., Naimzada, A., 1999. Symmetry-breaking bifurcations and representative firm in dynamic duopoly games, Annals of Operations Research 89, 253-272.

[7] Bischi, G.I., Stefanini, L., Gardini, L., 1998. Synchronization, intermittency and critical curves in a duopoly game. Mathematics and Computers in Simulation 44, 559-585.

[8] Brock, W.A., Hommes, C.H., 1997. A rational route to randomness. Econometrica 65, 1059-1095.

[9] Cánovas, J.S., Puu, T., Ruíz, M., 2008. The Cournot-Theocharis problem reconsidered. Chaos, Solitons \& Fractals 37, 1025-1039.

[10] Correa-López, M., Naylor, R.A., 2004. The Cournot-Bertrand profit differential: a reversal result in a differentiated duopoly with wage bargaining. European Economic Review 48, 681-696.

[11] Ellison, S.F., Snyder, C.M., 2014. An empirical study of pricing strategies in an online market with high-frequency price information. CESifo Working Paper no. 4655.

[12] Fanti, L., Gori, L., Sodini, M., 2012. Nonlinear dynamics in a Cournot duopoly with relative profit delegation. Chaos, Solitons \& Fractals 45, 1469-1478.

[13] Fanti, L., Gori, L., Sodini, M., 2013b. Nonlinear dynamics in a Cournot duopoly with different attitudes towards strategic uncertainty. Abstract and Applied Analysis 2013, Article ID 323290.

[14] Fanti, L., Gori, L., Sodini, M., 2015. Nonlinear dynamics in a Cournot duopoly with isoelastic demand. Mathematics and Computers in Simulation 108, 129-143.

[15] Fanti, L., Gori, L., Sodini, M., 2017. Managerial delegation theory revisited. Managerial and Decision Economics 38, 490-512.

[16] Fanti, L., Gori, L., Mammana, C., Michetti, E., 2013a. The dynamics of a Bertrand duopoly with differentiated products: synchronization, intermittency and global dynamics. Chaos, Solitons \& Fractals 52, 73-86.

[17] Gasmi, F., Laffont, J.J., Vuong, Q.H., 1992. Econometric analysis of collusive behavior in a soft-drink market. Journal of Economics and Management Strategy 1, 277-311.

[18] Naimzada, A.K., Tramontana, F., 2012. Dynamic properties of a Cournot-Bertrand duopoly game with differentiated products. Economic Modelling 29, 1436-1439.

[19] Newbery, D.M., Greve, T., 2015. The robustness of industrial commodity oligopoly pricing strategies. Cambridge Working Paper in Economics 1540. 
[20] Puu, T., 1991. Chaos in duopoly pricing. Chaos, Solitons \& Fractals 1, 573-581.

[21] Puu, T., 2017. A new approach to modeling Bertrand duopoly. Review of Behavioral Economics 4, 51-67.

[22] Singh, N., Vives, X., 1984. Price and quantity competition in a differentiated duopoly. RAND Journal of Economics 15, 546-554.

[23] Sushko, I., Gardini, L., Matsuyama, K., 2014. Superstable credit cycles and U-sequence. Chaos, Solitons \& Fractals 59, 13-27.

[24] Tramontana, F., Gardini, L., Puu, T., 2010. Global bifurcations in a piecewise-smooth Cournot duopoly game. Chaos, Solitons \& Fractals 43, 15-24.

[25] Varian, H.R., 1982. The nonparametric approach to demand analysis. Econometrica 50, 945-973.

[26] Varian, H.R., 1990. Goodness of fit in optimizing models. Journal of Econometrics 46, $125-140$.

[27] Wang, H., Ma, J., 2014. Complexity analysis of a Cournot-Bertrand duopoly game with different expectations. Nonlinear Dynamics 78, 2759-2768. 